\title{
Relativistic Hamiltonians for chemistry: a primer
}

\author{
Trond Saue* \\ Laboratoire de Chimie et Physique Quantique, UMR 5626 CNRS --- Université Toulouse III-Paul Sabatier, \\ 118 route de Narbonne, F-31062 Toulouse, France
}

A major breakthrough in relativistic quantum chemistry in recent years is the development of the exact 2-component (X2C) Hamiltonian which reproduces exactly the positive-energy spectrum of the parent 4-component Hamiltonian, yet is constructed in a simple manner using matrix algebra. This mini-review provides an overview of 4- and 2-component relativistic Hamiltonians employed in molecular electronic structure calculations and the underlying physics they carry, thus setting the recent developments in perspective.

* trond.saue@irsamc.ups-tlse.fr; http://dirac.ups-tlse.fr/saue 


\section{INTRODUCTION}

Relativity has added a new dimension to chemistry. Pople introduced the concept of theoretical model chemistries $[1,2]$ as specific combinations of molecular electronic structure methods and basis sets. Their formal independence means that the space of theoretical model chemistries may be spanned by two orthogonal axis representing the choice of method and basis. In practice balanced combinations of these two are sought, preferably with the possibility of a systematic approach towards exact solutions of the underlying wave equation. Increased accuracy of a model chemistry generally comes with increased computational cost, which can be expressed as $N^{y}$, where $N$ is the basis set size and $y$ a method-dependent power. A major undertaking in the past few decades has been to bring the computational cost down towards linear scaling with respect to system size [3]. However, it has also become increasingly clear that the applicability of theoretical model chemistries for the study of chemistry involving elements across the entire periodic table requires that relativistic effects are taken into account [4]. Such effects arise from the high speed of electrons in the vicinity of heavy nuclei. One distinguishes between scalar relativistic effects, associated with the relativistic mass increase of electrons, and the spin-orbit interaction, generated by magnetic induction, a mechanism that can not be described within a non-relativistic framework [5].

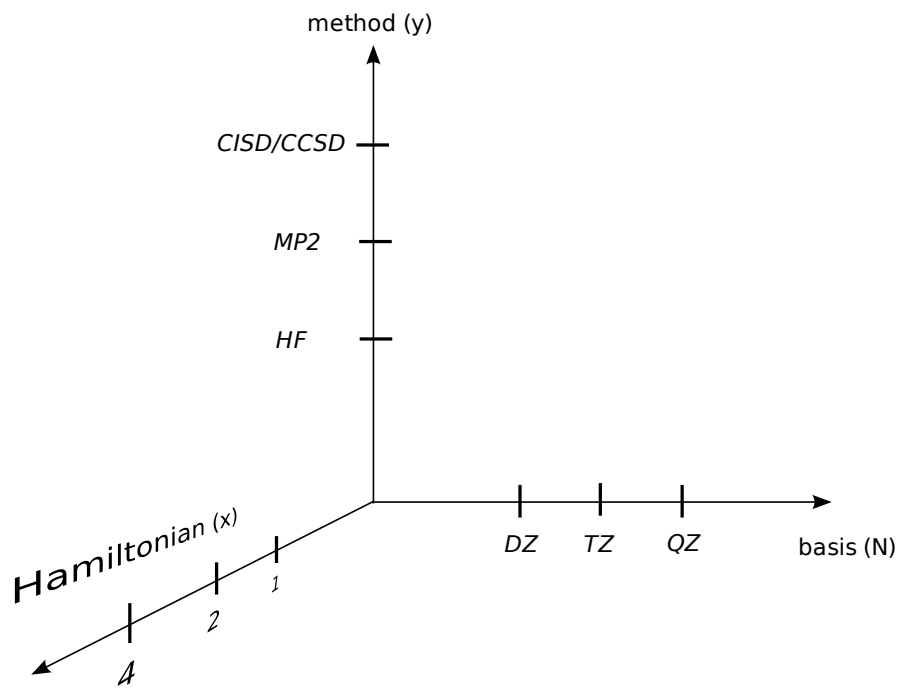

Figure 1. A 3D chart of theoretical model chemistries

Relativistic effects are introduced through the choice of Hamiltonian. There are many relativistic Hamiltonians on the market, but they can all be defined with respect to the generic form of the electronic Hamiltonian within the Born-Oppenheimer approximation

$$
H=\sum_{i} \hat{h}(i)+\frac{1}{2} \sum_{i \neq j} \hat{g}(i, j)+V_{N N},
$$

where $V_{N N}$ is the classical repulsion of clamped nuclei. The basic formulas of present molecular electronic structure methods can be developed without specification of the one-electron operator $\hat{h}$ and the two-electron operator $\hat{g}$, which implies that the various non-relativistic methods available today can be carried over into the relativistic domain, albeit with possible adaptions. The choice of Hamiltonian introduces accordingly a third axis for the specification of theoretical model chemistries [6], as depicted in figure 1. The independence of the choice of method and of Hamiltonians is often muddled in relativistic terminology where for instance Dirac-Hartree-Fock is presented as a method, whereas it refers to a specific choice of both method and Hamiltonian. The shortened form Dirac-Fock is in addition unfair because Bertha Swirles made the first formulation of 4-component relativistic Hartree-Fock theory following a suggestion by Douglas Hartree [7].

A convenient specification of Hamiltonians along this axis is with respect to the dimension of the one-electron operator. The non-relativistic one-electron operator is a scalar operator, whereas the fully relativistic Dirac Hamiltonian is a $4 \times 4$ matrix operator. The increased dimension of the corresponding one-electron wave functions (orbitals) is due to the fact that the Dirac equation explicitly includes spin and describes two kinds of particles: the electron and its anti-particle, the positron, having an entangled existence.

In between the scalar non-relativistic and 4-component relativistic one-electron Hamiltonians there are a number of 2-component relativistic Hamiltonians where the positronic degrees of freedom have been frozen. Whereas scalar 
relativistic effects may be introduced into a non-relativistic computer code with essentially no extra computational cost, the description of the spin-orbit interaction requires at least a 2-component formalism and will increase computational cost, typically by one order of magnitude, due to the transition from real to complex algebra and the general reduction of symmetry. Full relaxation of the electronic wave function is only possible at the 4-component level, as will be discussed in section IIC, but formally increases the computational cost by another order of magnitude. It is, however, important to stress that the additional computational effort associated with the introduction of relativistic Hamiltonians is a prefactor and thus independent of system size.

In the past few years there has been significant excitement in the domain of (relativistic) quantum chemistry due to the development of the so-called exact 2-component relativistic (X2C) Hamiltonian which reproduces exactly the positive-energy spectrum of the parent 4-component Hamiltonian. Recently Wenjian Liu, one of the key players in the field, published a comprehensive review on 2-component Hamiltonians [8]. The review is rather complete and full of insight, but also quite technical and easily gives the impression that the construction of the X2C Hamiltonian is complicated. This has motivated me to write the present mini-review which is a more introductory text. Since I strongly believe that it is important to add some flesh in terms of physics to the skeleton of theoretical expressions I start with a discussion of 4-component Hamiltonians. I also hope to clear up some misunderstandings in the literature and share some insight gained over more than twenty years of working with relativistic molecular quantum mechanics. I will furthermore discuss some 2-component approximate relativistic Hamiltonians: i) the Pauli Hamiltonian, because its underlying physics is particularly transparent, and ii) the Zeroth-Order Regular Approximation (ZORA) and Douglas-Kroll-Hess (DKH) Hamiltonians, because they are widely used. I then turn to a discussion of the X2C Hamiltonian, where I will stress the simplicity of its formulation. I hope to convince the reader of this by describing in some detail the one-step construction of the X2C Hamiltonian as implemented by Miroslav Iliaš in the DIRAC code [9] and recently re-written in more modular form by Stefan Knecht. It should not be forgotten that the 2-component Hamiltonians are typically obtained by some decoupling transformation of a parent one-electron Hamiltonian, and so the proper handling of the two-electron interaction as well as property operators merits separate discussions. Next, I will give a numerical illustration, where all results have been obtained with the same computer code and the same basis set, to illustrate the performance of the various Hamiltonians discussed in the text. Finally, I will discuss what has been gained by the introduction of the X2C Hamiltonian, and also what are its possible disadvantages. I will conclude by a personal outlook on the future of 2-component relativistic Hamiltonians and of relativistic quantum chemistry in general.

The present contribution is a primer and accordingly willingly brief and limited in scope. I hope to leave sufficient references for the interested reader to continue exploring this fascinating domain of theoretical chemistry. There are also some excellent textbooks available. In the molecular domain I would recommend the book by Kenneth Dyall and Knut Fægri [10] as well as the book by Markus Reiher and Alexander Wolf [11], which are to some extent complementary. Peter Schwerdtfeger has recently edited a collection of contributions on the fundamentals and applications of relativistic electronic structure theory $[12,13]$. I can also recommend similar compilations by M. Barysz and Y. Ishikawa [14] as well as U. Kaldor and S. Wilson [15]. A clear omission in the present mini-review are relativistic pseudopotentials, for which excellent reviews are available [16-19].

Unless otherwise stated I employ SI-based atomic units [20] in the following.

\section{4-COMPONENT HAMILTONIANS}

\section{A. One-electron part}

We start from the classical expression for the energy of a relativistic free particle

$$
E^{2}=m^{2} c^{4}+c^{2} p^{2}
$$

where $m$ is mass, $c$ the speed of light and $p$ linear momentum. Taking the square root on each side of the above expression we are faced with a choice of sign

$$
E= \pm \sqrt{m^{2} c^{4}+c^{2} p^{2}} ; \quad \Rightarrow \quad E \in\left\langle-\infty,-m c^{2}\right] \cup\left[+m c^{2}, \infty\right\rangle
$$

showing that possible values of the energy $E$ lie in two bands of opposite sign separated by a huge energy gap of $2 m c^{2}$. In classical mechanics we can safely discard the negative energy branch as unphysical since the energy can only change in a continuous manner. This is, however, not possible in quantum mechanics, where quantum leaps in energy are, as the name implies, possible.

The relativistic energy expression does not resemble its non-relativistic counterpart

$$
E^{N R}=\frac{p^{2}}{2 m}
$$


at all, but the two can be connected by a Taylor expansion of the positive-energy branch of the former

$$
E=m c^{2} \sqrt{1+\frac{p^{2}}{m^{2} c^{2}}}=\underbrace{m c^{2}}_{\text {rest mass }}+\underbrace{\frac{p^{2}}{2 m}-\frac{p^{4}}{8 m^{3} c^{2}}+\ldots}_{\text {kinetic energy }}
$$

The first term is the rest mass, the second term is the energy of a non-relativistic free particle, whereas further terms are relativistic corrections that go to zero as $c \rightarrow \infty$. Since there is no absolute origin of the energy scale, we can align the relativistic scale with the non-relativistic one by subtracting the rest mass $E \rightarrow E^{+}=E-m c^{2}$. For negative-energy solutions, on the other hand, the proper change of energy scale in order to obtain finite energies in the non-relativistic limit is $E \rightarrow E^{-}=E+m c^{2}$.

The quantization of the non-relativistic energy expression (4) is straightforward: We replace the coordinate and momentum variables by the corresponding operators, perform the heuristic substitution $E \rightarrow i \frac{\partial}{\partial t}$ and insert a wave function on both sides to obtain the Schrödinger equation for a free particle. The relativistic case is quite a bit more complicated and was not handled successfully until Dirac obtained a linearisation of the energy expression (2) [21, 22]. Here show an alternative derivation due to van Waerden [23]. It is a useful exercise since it provides an introduction to the use of the Dirac identity

$$
(\boldsymbol{\sigma} \cdot \mathbf{A})(\boldsymbol{\sigma} \cdot \mathbf{B})=\mathbf{A} \cdot \mathbf{B}+i \boldsymbol{\sigma} \cdot(\mathbf{A} \times \mathbf{B}) .
$$

involving the three Pauli spin matrices collected in the vector $\sigma$. A particular instance of this relation is

$$
(\boldsymbol{\sigma} \cdot \hat{\mathbf{p}})(\boldsymbol{\sigma} \cdot \hat{\mathbf{p}})=p^{2}
$$

suggesting that spin may be "hidden" in the non-relativistic wave equation and only comes into play when an external magnetic field is introduced. In the following we shall drop the hats indicating operators.

A first step towards the Dirac equation is to re-arrange the relativistic energy expression (2) and to perform quantization as discussed above

$$
\left(-\frac{1}{c^{2}} \frac{\partial^{2}}{\partial t^{2}}-p^{2}\right) \phi_{1}=\left[\frac{i}{c} \frac{\partial}{\partial t}+(\boldsymbol{\sigma} \cdot \mathbf{p})\right] \underbrace{\left[\frac{i}{c} \frac{\partial}{\partial t}-(\boldsymbol{\sigma} \cdot \mathbf{p})\right] \phi_{1}}_{m c \phi_{2}}=(m c)^{2} \phi_{1} .
$$

Factorization is obtained through the use of the Dirac relation (6), more precisely (7), which implies that the wave function $\phi_{1}$ is a two-component vector function. We next introduce a second wave function $\phi_{2}$ to obtain two coupled equations

$$
\begin{aligned}
& {\left[\frac{i}{c} \frac{\partial}{\partial t}-(\boldsymbol{\sigma} \cdot \mathbf{p})\right] \phi_{1}=m c \phi_{2} \quad(a)} \\
& {\left[\frac{i}{c} \frac{\partial}{\partial t}+(\boldsymbol{\sigma} \cdot \mathbf{p})\right] \phi_{2}=m c \phi_{1}}
\end{aligned}
$$

As a third step we take linear combinations

$$
\begin{aligned}
& \frac{i}{c} \frac{\partial}{\partial t}\left[\phi_{1}+\phi_{2}\right]-(\boldsymbol{\sigma} \cdot \mathbf{p})\left[\phi_{1}-\phi_{2}\right]=m c\left[\phi_{1}+\phi_{2}\right] \quad(a+b) \\
& -\frac{i}{c} \frac{\partial}{\partial t}\left[\phi_{1}-\phi_{2}\right]+(\boldsymbol{\sigma} \cdot \mathbf{p})\left[\phi_{1}+\phi_{2}\right]=m c\left[\phi_{1}-\phi_{2}\right](b-a)
\end{aligned}
$$

and introduce the large $\psi^{L}$ and small $\psi^{S}$ components

$$
\psi^{L}=\left[\phi_{1}+\phi_{2}\right] ; \quad \psi^{S}=\left[\phi_{1}-\phi_{2}\right] .
$$

The resulting equations can be recast on matrix form

$$
\left[\begin{array}{cc}
\frac{i}{c} \frac{\partial}{\partial t} & -(\boldsymbol{\sigma} \cdot \mathbf{p}) \\
(\boldsymbol{\sigma} \cdot \mathbf{p}) & -\frac{i}{c} \frac{\partial}{\partial t}
\end{array}\right]\left[\begin{array}{l}
\psi^{L} \\
\psi^{S}
\end{array}\right]=m c\left[\begin{array}{l}
\psi^{L} \\
\psi^{S}
\end{array}\right]
$$

and the conventional form of the Dirac equation

$$
\left[\beta m c^{2}+c(\boldsymbol{\alpha} \cdot \mathbf{p})\right] \psi=i \frac{\partial}{\partial t} \psi ; \quad \psi=\left[\begin{array}{c}
\psi^{L} \\
\psi^{S}
\end{array}\right]
$$

is obtained by multiplication by $\beta c$ from the left, where we have introduced the Dirac matrices

$$
\boldsymbol{\alpha}=\left[\begin{array}{cc}
0_{2} & \boldsymbol{\sigma} \\
\boldsymbol{\sigma} & 0_{2}
\end{array}\right], \quad \beta=\left[\begin{array}{cc}
I_{2} & 0_{2} \\
0_{2} & -I_{2}
\end{array}\right]
$$


As in the non-relativistic case we may separate off the time-dependent part to obtain the Dirac equation on timeindependent form, albeit specific for a given inertial frame.

The free-particle Dirac equation is admittedly not very useful for chemistry and we therefore proceed to the introduction of external fields in accordance with the principle of minimal electromagnetic coupling [24]

$$
\mathbf{p} \rightarrow \mathbf{p}-q \mathbf{A} ; \quad E \rightarrow E-q \phi
$$

where appears particle charge $q$. The Dirac equation describes both electrons and positrons, having opposite charge. As a first step towards chemistry we focus on electrons and set $q=-e$, where $e$ is the fundamental charge. Note, however, that this implies that all solutions, of both positive and negative energy, of the resulting Dirac equation for the given potentials are electronic. The positronic solutions of the same external fields are related to the electronic ones through charge conjugation. For use in the calculation of the electronic structure of molecules we shall invoke the Born-Oppenheimer (clamped nuclei) approximation. We furthermore limit attention to nuclear charge, introducing the notation $V=-e \phi_{n u c}$, where $\phi_{\text {nuc }}$ is the electrostatic potential of clamped nuclei. The effect of nuclear spin is much weaker and can be treated by perturbation theory, as in the calculation of NMR parameters. We accordingly arrive at the Dirac equation for an electron in a molecular field

$$
h_{D} \psi=E^{+} \psi ; \quad h_{D}=\beta^{\prime} m c^{2}+c(\boldsymbol{\alpha} \cdot \mathbf{p})+V=\left[\begin{array}{cc}
V & c(\boldsymbol{\sigma} \cdot \mathbf{p}) \\
c(\boldsymbol{\sigma} \cdot \mathbf{p}) & V-2 m c^{2}
\end{array}\right] ; \quad \beta^{\prime}=\beta-I_{4}
$$

where the relativistic energy scale has been aligned with the non-relativistic one, as discussed above.

The so-called minimal substitution (15) is employed both in the relativistic and non-relativistic domain, but it is important to realize that it corresponds to a relativistic coupling of particles and fields [5, 25]. Non-relativistic theory is notably unable to describe magnetic induction, which is a relativistic effect and forms the basis for the spin-orbit interaction. Figure 2 illustrates the basic mechanism of spin-orbit interaction generated by a clamped nucleus of charge $+Z e$. In the frame of the nucleus, which is the frame employed for the formulation of the electronic Hamiltonian, the nucleus is a source of a electrostatic potential $\phi$ only and the vector potential $\boldsymbol{A}$ is zero. However, carrying out a Lorentz transformation to the frame of the moving electron a non-zero vector potential appears, meaning that the electron in its own frame sees both an electric and magnetic field due to the nucleus in relative motion. Spin-orbit coupling is the interaction of the electron spin with the magnetic field induced by a charge in relative motion. A subtle feature of the spin-orbit interaction arises from the fact that the motion of the electron is not of constant velocity and thus has to be followed by a succession of Lorentz transformations. The product of two pure Lorentz transformations (boosts) is not a third boost, rather a boost combined with a rotation [26], so that the succession of boosts induces a rotational motion, denoted the Thomas precession, which reduces the spin-orbit interaction by a factor two.

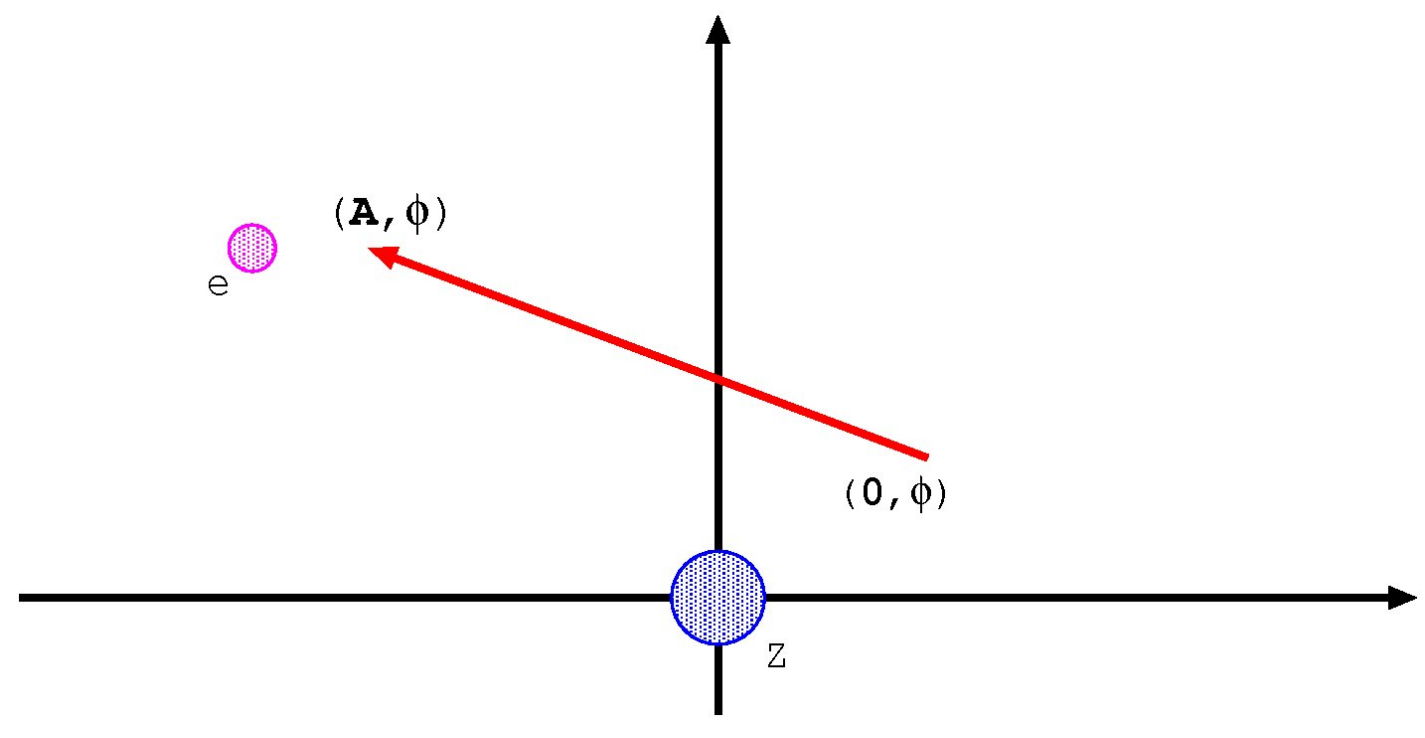

Figure 2. Basic mechanism of spin-orbit interaction generated by a clamped nucleus. The arrow represents a Lorentz transformation from the frame of clamped nuclei to the frame of the moving electron. 


\section{B. Two-electron part}

The derivation of the relativistic expression for the two-electron interaction can be seen as a continuation of the preceding discussion in that we replace the potentials of a clamped nucleus by those of a second moving electron, taking care of avoiding double counting of interactions [27]. In Coulomb gauge the general expression for the scalar potential is

$$
\phi\left(\mathbf{r}_{1}, t\right)=\int \frac{\rho\left(\mathbf{r}_{2}, t\right)}{r_{12}} d \tau_{2} ; \quad r_{12}=\left|\boldsymbol{r}_{1}-\boldsymbol{r}_{2}\right|
$$

which corresponds to the instantaneous Coulomb interaction. The complexity of the relativistic two-electron interaction shows up in the expression for the corresponding vector potential

$$
\mathbf{A}\left(\mathbf{r}_{1}, t\right)=\frac{4 \pi}{c^{2}} \int \frac{\mathbf{j}_{\perp}\left(\mathbf{r}_{2}, t_{r}\right)}{r_{12}} d \tau_{2} ; \quad t_{r}=t-\frac{r_{12}}{c},
$$

where $\mathbf{j}_{\perp}$ is the solenoidal, or divergence-free, current density $\left(\boldsymbol{\nabla} \cdot \mathbf{j}_{\perp}=0\right)$, compatible with the constraint of a purely solenoidal vector potential $\left(\boldsymbol{A}=\boldsymbol{A}_{\perp}\right)$ in Coulomb gauge. Contrary to the expression for the scalar potential we now have retarded time $t_{r}$, and not instantaneous time $t$, under the integral sign, reflecting the finite speed of the propagation of interactions in the relativistic domain. For classical electrons we accordingly need to know the position $\boldsymbol{r}_{2}$ of the second electron at retarded time. In fact, the full history of the interacting particles is required for a complete relativistic description of the two-electron interaction, and no closed expression is available for use in the electronic Hamiltonian. Rather a perturbation expansion of the full two-electron interaction can be used, starting from the instantaneous Coulomb term known from the non-relativistic domain

$$
g^{C}(1,2)=\frac{e^{2}}{r_{12}} .
$$

We can indeed consider the instantaneous Coulomb interaction as the non-relativistic limit of electrodynamics, as suggested by the observation the the vector potential (18) goes to zero in the non-relativistic limit. The question of the non-relativistic limit of electrodynamics is to some extent open, since there is no experiment which can probe this limit. It has been suggested that magnetic induction, e.g. the spin-orbit interaction, goes to zero in the non-relativistic limit, but not the magnetic fields themselves [28, 29]. However, this leads to incoherent theory, as shown by a simple thought experiment: Suppose that we consider a system consisting of a molecule to which we apply an external magnetic field. If we now extend the system to include the sources of the magnetic field, then the magnetic field will vanish in the non-relativistic limit for the extended system, but not for the original one. A more consistent proposal is that all magnetic interactions as well as retardation disappears in the non-relativistic limit [5, 30]. A non-relativistic framework can certainly accommodate magnetic fields, but this amounts to a non-relativistic description of particles and a relativistic description of their coupling to electromagnetic fields.

We can derive the first-order relativistic correction to the two-electron interaction in a heuristic manner by starting from the classical Darwin approximation to the relativistic interaction between two moving charges[31]

$$
E_{\text {int }}=q_{1} \phi_{2}-q_{1} \mathbf{v}_{1} \cdot \mathbf{A}_{2} \sim \frac{q_{1} q_{2}}{r_{12}}-\frac{q_{1} \mathbf{v}_{1} \cdot q_{2} \mathbf{v}_{2}}{2 c^{2} r_{12}}-\frac{\left(q_{1} \mathbf{v}_{1} \cdot \mathbf{r}_{12}\right)\left(q_{2} \mathbf{v}_{2} \cdot \mathbf{r}_{12}\right)}{2 c^{2} r_{12}^{3}} .
$$

The first term on the far right is, as expected, the Coulomb term, whereas the final two terms involve the vector potential of the second electron and come in to order $O\left(c^{-2}\right)$. For quantization we shall need the relativistic velocity operator which can be obtained from the Heisenberg equation of motion

$$
\frac{d \mathbf{r}}{d t}=-i\left[\mathbf{r}, h_{D}\right]=c \boldsymbol{\alpha} .
$$

The form of this operator is quite different from the non-relativistic velocity operator $\frac{p}{m}$ and reflects Zitterbewegung [32], a highly oscillatory motion superimposed on the average motion of the electron. We obtain the Breit term

$$
g^{B}(1,2)=-e^{2}\left\{\frac{c \boldsymbol{\alpha}_{1} \cdot c \boldsymbol{\alpha}_{2}}{2 c^{2} r_{12}}+\frac{\left(c \boldsymbol{\alpha}_{1} \cdot \mathbf{r}_{12}\right)\left(c \boldsymbol{\alpha}_{2} \cdot \mathbf{r}_{12}\right)}{2 c^{2} r_{12}^{3}}\right\}
$$

by the heuristic substitution $v_{i} \rightarrow c \boldsymbol{\alpha}_{i}$ in (20), which is indeed how Breit obtained this operator himself [33]. The Breit term can be re-arranged to [34]

$$
g^{B}(1,2)=g^{G}(1,2)+g^{\text {gauge }}(1,2)=-\frac{e c \boldsymbol{\alpha}_{1} \cdot e c \boldsymbol{\alpha}_{2}}{c^{2} r_{12}}-\frac{\left(e c \boldsymbol{\alpha}_{1} \cdot \nabla_{1}\right)\left(e c \boldsymbol{\alpha}_{2} \cdot \nabla_{2}\right) r_{12}}{2 c^{2}},
$$




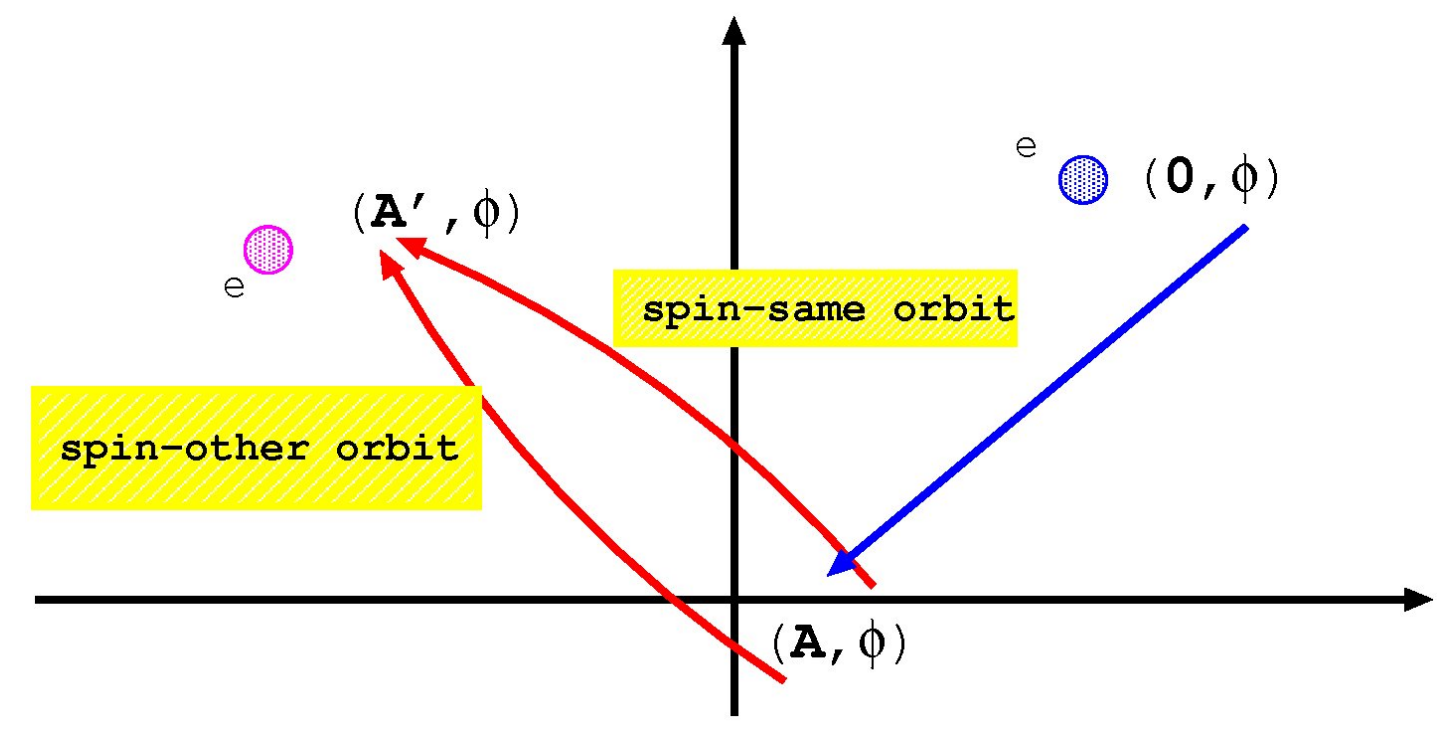

Figure 3. Basic mechanism of spin-orbit interaction between electrons. The arrows represents Lorentz transformations between the frame of the moving electrons and the frame of clamped nuclei.

where the first term is known as the Gaunt term [35] and represents current-current interaction, contrary to the Coulomb term which represents charge-charge interaction.

Both terms give rise to spin-orbit interaction. The separation of spin-orbit interaction due to relative motion of electrons into two terms, contrary to the single term associated with electron-nucleus interaction, is due to the choice of reference frame for the specification of the electronic Hamiltonian, as illustrated in figure 3 . The second electron contributes only a scalar potential in its own frame, just as the the clamped nuclei in the Born-Oppenheimer frame. However, Lorentz transforming to the frame of the nuclei a non-zero vector potential appears, which gives rise to the Gaunt (Breit) term. Both the scalar and vector potential of the second electron, as seen in the Born-Oppenheimer frame, contribute to the vector potential in the frame of the reference electron and give rise to the spin-same-orbit (SSO) and spin-other-orbit (SOO) interactions, respectively. The spin-other-orbit interaction arises solely from the Gaunt term and not from the gauge-dependent term $g^{\text {gauge }}(1,2)[36]$. From these considerations we see that the spinorbit interaction associated with nuclei is of the spin-same-orbit type. Finally, it should be noted that the Lorentz transformation of the instantaneous Coulomb term to some other frame does in general not give potentials satisfying Coulomb gauge, but this can be achieved by carrying out the proper gauge transformation in the new frame.

\section{The electronic Hamiltonian}

Most 4-component relativistic molecular calculations are based on the Dirac-Coulomb Hamiltonian, where the Dirac Hamiltonian $h_{D}$ in the molecular field (16) is combined with the Coulomb two-electron operator (19). Although the latter operator has essentially the same form as in the non-relativistic domain, its physical content is different in that it includes spin-same orbit (SSO) interaction, as discussed in section IIB. The truncation of the full two-electron interaction means that the electronic Hamiltonian is not strictly relativistic, but it is sufficient for most chemical purposes [37]. For accurate studies of molecular spectra including fine structure it is recommended to include spinother orbit interaction through the Gaunt (Breit) term. The Gaunt term also carries the full spin-spin interaction, whereas the gauge-dependent term $g^{\text {gauge }}$ (23) must be included for the full orbit-orbit interaction [36].

The first 4-component relativistic Hartree-Fock calculations based on the Dirac-Coulomb Hamiltonian and using finite basis sets [38-40] were flawed since the coupling of large and small components were not taken into account [41-44]. From the Dirac equation for an electron in a molecular field (16) the exact coupling is found to be

$$
\psi^{S}=R \psi^{L} ; \quad R(E)=\left(2 m c^{2}-V+E^{+}\right)^{-1} c(\boldsymbol{\sigma} \cdot \mathbf{p})=\frac{1}{2 m c} B(E)(\boldsymbol{\sigma} \cdot \mathbf{p}) ; \quad B(E)=\left[1+\frac{E^{+}-V}{2 m c^{2}}\right]^{-1} .
$$

For positive-energy solutions the energy-dependent factor $B(E)$ is on the order of unity $O\left(c^{0}\right)$, demonstrating that the small components are on average a factor $c$ smaller than the large components, hence their name. For negative-energy 
solutions $B(E)$ can be re-expressed as

$$
B(E)=\frac{2 m c^{2}}{E^{-}-V}
$$

and is of order $O\left(c^{2}\right)$, such that the relative sizes of the large and small components switch. Due to the energy dependence of the exact coupling (24) one rather employs its non-relativistic limit

$$
\lim _{c \rightarrow \infty} c \psi^{S}=\frac{1}{2 m}(\boldsymbol{\sigma} \cdot \mathbf{p}) \psi^{L} .
$$

Note, however, that this limit is only valid for $\left|E^{+}-V\right| \ll 2 m c^{2}$ which restricts the energy to the positive-energy branch, for which $E^{+} \sim O\left(c^{0}\right)$, and the potential $\phi_{n u c}$ to non-singular ones, the latter obtained by replacing the point charge nuclear model of the non-relativistic domain by a finite charge distribution. A further strong motivation for the introduction of a finite nuclear charge distribution is that a point nucleus induces a singularity in the electronic wave function at the nucleus, and not a cusp as in the non-relativistic domain. Although the singularity is weak in the sense that it still allows normalization of the wave function, it can not be described in a finite basis using conventional Slater- or Gaussian-type functions and serves as a 'black hole' in basis set optimizations in that it can not be saturated by the introduction of tight functions. With the introduction of a finite nuclear charge model, typically a Gaussian, the singularity is replaced by a Gaussian shape at the origin, favoring the use of Gaussian-type basis functions [45-47].

The implementation of (26) at the basis set level has been denoted kinetic balance [48], since it provides a correct representation of the kinetic energy operator in the non-relativistic limit. It is, however, important to have sufficient flexibility in the basis such that the exact coupling can be obtained [49]. The effect of the missing energy-dependent factor $B(E)$ is illustrated in figure 4 which shows the small component radial function $R^{S}$ of the $1 s_{1 / 2}$ orbital of the mercury atom obtained by a numerical atomic 4-component relativistic Hartree-Fock calculation (exact coupling) or generated from the large component radial function $R^{L}$ using restricted kinetic balance. In this particular case $B(E)$ can be expanded as

$$
B\left(\varepsilon_{1 s}\right)=1+\frac{\left|\varepsilon_{1 s}\right|}{2 m c^{2}}-\frac{e \phi_{n u c}}{2 m c^{2}}+\ldots
$$

where $\varepsilon_{1 s}$ is the $1 s_{1 / 2}$ orbital energy. A finite (Gaussian) nucleus model was used in this calculation such that there is no singularity of the corresponding potential $\phi_{n u c}$ at the origin. Yet the potential attains significant values close to the nucleus and the $B(E)$ factor will accordingly dampen the RKB radial functional in this region, as seen in the figure. However, bearing in mind that the radial expectation value of the $1 s_{1 / 2}$ orbital is $0.0017 a_{0}$ it can be seen that this modification is extremely local, which implies that if the exact coupling can be obtained by a kinetically balanced basis for an isolated atom it will also work when this atom is placed in a molecule [50]. Eq. (27) furthermore suggests that away from the nucleus, for sufficiently small $\phi_{n u c}$, the factor $B\left(\varepsilon_{1 s}\right)$ will be slightly larger than unity.

In a finite basis calculation the modification of the coupling of large and small components from kinetic to exact balance is carried by the basis set expansion coefficients, which commute with the operator $(\boldsymbol{\sigma} \cdot \boldsymbol{p})$. It has therefore been pointed out [52-54] that imposing kinetic balance is equivalent to transforming the Dirac equation (16) according to

$$
\left[\begin{array}{l}
\psi^{L} \\
\psi^{S}
\end{array}\right]=\left[\begin{array}{cc}
I_{2} & 0_{2} \\
0_{2} & \frac{1}{2 m c}(\boldsymbol{\sigma} \cdot \boldsymbol{p})
\end{array}\right]\left[\begin{array}{c}
\psi^{L} \\
\phi^{L}
\end{array}\right]
$$

where $\phi^{L}$ is denoted the pseudo-large component. The resulting equation

$$
\left[\begin{array}{cc}
V & T \\
T & W_{0}-T
\end{array}\right]\left[\begin{array}{c}
\psi^{L} \\
\phi^{L}
\end{array}\right]=\left[\begin{array}{cc}
I_{2} & 0_{2} \\
0_{2} & \frac{1}{2 m c^{2}} T
\end{array}\right]\left[\begin{array}{c}
\psi^{L} \\
\phi^{L}
\end{array}\right] E ; \quad T=\frac{p^{2}}{2 m} ; \quad W_{0}=\frac{1}{4 m^{2} c^{2}}(\boldsymbol{\sigma} \cdot \boldsymbol{p}) V(\boldsymbol{\sigma} \cdot \boldsymbol{p})
$$

was later revived and denoted the modified Dirac equation by Dyall [53], who used it to obtain a spin-orbit free form of the Dirac equation. This is accomplished by using the Dirac identity (6)

$$
(\boldsymbol{\sigma} \cdot \boldsymbol{p}) V(\boldsymbol{\sigma} \cdot \boldsymbol{p})=\boldsymbol{p} V \cdot \boldsymbol{p}+i \boldsymbol{\sigma} \cdot(\boldsymbol{p} V \times \boldsymbol{p})
$$

and remove the second term. In a quaternion formalism [55] this corresponds to the deletion of imaginary parts of the quaternion modified Dirac equation. Visscher and van Lenthe suggested that the separation of spin-free and spindependent terms is not unique[56]. This is only partially true. Going back to figure 2 we see that a precise mechanism for the elimination of the spin-orbit interaction would be to eliminate the vector potential of the nucleus in the rest frame of the electron. The transformation back to the nuclear frame is equivalent to the forward transformation with 


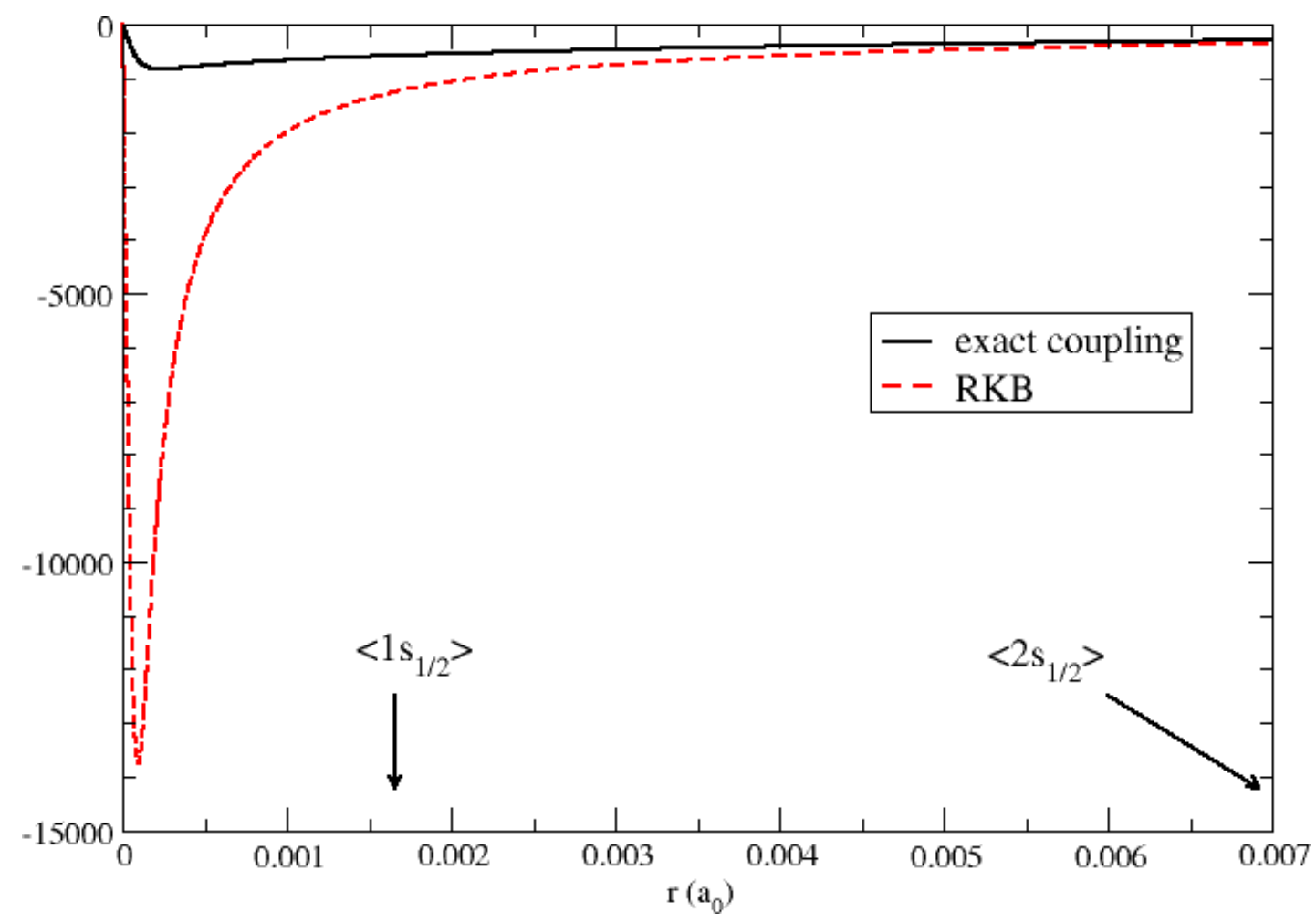

Figure 4. The small component $1 s$ radial function of the mercury atom (finite Gaussian nucleus) generated from the large component using exact coupling or restricted kinetic balance. Radial expectation values of the $1 s_{1 / 2}$ and $2 s_{1 / 2}$ orbitals are indicated for reference. The data is based on a numerical 4-component relativistic Hartree-Fock calculation using the GRASP code [51].

the opposite sign of the velocity and would therefore result in the introduction of a compensating magnetic field in the nuclear frame. However, there is no practical realization of such a procedure.

Another interesting feature of the modified Dirac equation is that, contrary to the parent equation (16), it contains only inverse powers of the speed of light such that the non-relativistic limit can be taken without the operator blowing up. The non-relativistic limit of the Dirac equation can also be obtained by the simple metric transformation

$$
\left[\begin{array}{c}
\psi^{L} \\
\psi^{S}
\end{array}\right]=\left[\begin{array}{cc}
I_{2} & 0_{2} \\
0_{2} & I_{2} c^{-1}
\end{array}\right]\left[\begin{array}{c}
\psi^{L} \\
\tilde{\psi}^{S}
\end{array}\right]
$$

which leads to the equation

$$
\left[\begin{array}{cc}
V & (\boldsymbol{\sigma} \cdot \mathbf{p}) \\
(\boldsymbol{\sigma} \cdot \mathbf{p}) & -2 m\left(1-\frac{V}{2 m c^{2}}\right)
\end{array}\right]\left[\begin{array}{c}
\psi^{L} \\
\widetilde{\psi}^{S}
\end{array}\right]=\left[\begin{array}{cc}
I_{2} & 0_{2} \\
0_{2} & c^{-2} I_{2}
\end{array}\right]\left[\begin{array}{c}
\psi^{L} \\
\widetilde{\psi}^{S}
\end{array}\right] E
$$

for which the non-relativistic limit is known as the Lévy-Leblond equation [57]. Note, however, that in both cases it is assumed that $E \ll 2 m c^{2}$ and $V \ll 2 m c^{2}$, which is only true for positive-energy solutions and extended nuclei, respectively. Equation (32) is the starting point for direct perturbation theory (DPT) [28, 58-60] which has recently been revived by Stopkowich and co-workers [61-63].

The Dirac-Coulomb Hamiltonian has no bound solutions. Expanding the many-electron wave function in an Nparticle basis of Slater determinants one finds that a determinant of bound orbitals will be degenerate with an infinite number of determinants containing orbitals from both the positive- and negative-energy continuum. These determinants will mix in and cause a continuum dissolution[64], referred to as the Brown-Ravenhall disease. It can be avoided by embedding the Dirac-Coulomb Hamiltonian by projection operators removing any Slater determinant containing negative-energy orbitals [65]. Such a no-pair Hamiltonian is simply obtained by expressing the electronic Hamiltonian in second quantization

$$
\hat{H}=\sum_{p q} h_{p q} a_{p}^{\dagger} a_{q}+\frac{1}{2} \sum_{p q r s} g_{p q r s} a_{p}^{\dagger} a_{r}^{\dagger} a_{s} a_{q}
$$


and removing all negative-energy orbitals from the summations. A crucial point and a source of much confusion in the literature is that the separation of the space spanned separately by positive- and negative-energy orbitals depends on the actual potential such that projectors can not be introduced without carefully specifying the potential on which they are based.

The one-electron basis for which the N-particle basis is constructed is generated by solving some effective oneelectron Dirac equation, typically the Hartree-Fock equation. Such an equation has, just as the Dirac equation itself, solutions of both positive and negative energy. The bound orbitals employed for the construction of the mean-field potential are not selected according to an Aufbau principle, starting from the orbitals of lowest energy. Rather one starts from the lowest positive-energy orbital, which is readily identified due to the large energy gap, on the order of $2 m c^{2}$, down to the negative-energy solutions. This procedure corresponds to the implicit use of projection operators updated in every iteration of the SCF procedure, as suggested by Mittleman [66]. It furthermore corresponds to the variational solution of the Dirac equation, replacing the minimization principle of the non-relativistic domain by a min-max principle[67, 68]. The original proposal by Sucher[65] was to employ projection operators constructed from the solutions of the free-particle Dirac equation. However, as pointed out by Heully and co-workers[69], this will induce serious errors. A better approximation to the mean-field projectors is to use the bare nucleus projectors, that is, using the solutions of the Dirac equation in the molecular field (16), which, as we shall see in section III, are implicitly used in most schemes for generating 2-component relativistic Hamiltonians.

Starting from the Hartree-Fock solutions, electron correlation may be added through Configuration Interaction (CI) or Coupled Cluster (CC) procedures. The N-particle determinants are usually constructed from positive-energy orbitals only. This bars, however, the complete relaxation of the one-particle basis and thus the projectors to the full instantaneous potential seen by the electrons of the system and implies that a full CI in this determinantal space can not be considered as the exact solution of the optimal projected Dirac-Coulomb Hamiltonian. Various authors[70-72] have reported CI calculations using an N-particle basis constructed from both positive- and negative-energy orbitals. Pestka and co-workers[73] have reported a procedure in which the electronic ground state is treated as a resonance and extracted from the continuum by a complex-coordinate rotation technique. However, in all such procedures going beyond the no-pair approximation the electronic ground state is therefore at best treated as a metastable state. Ultimately this would imply that matter is not stable, which is physically not very satisfying. In the early days of the Dirac equation the stability of matter caused considerable worry amongst theoreticians since calculations based on the Dirac equation suggested that the hydrogen atom would have a lifetime of about a nanosecond, after which the electron would descend down the negative-energy continuum, liberating an infinite amount of energy. Dirac finally proposed that all negative-energy orbitals are occupied and thus not available. A more satisfying treatment of the negative-energy states is obtained in the framework of QED which treat electrons and positrons on an equal footing.

Present-day relativistic molecular electronic structure calculations treat the negative-energy orbitals as an orthogonal complement which is continuously updated at the SCF level and frozen at the correlated level. A complete relaxation of the electronic wave function is possible at the MCSCF level with the N-particle basis constructed according to the no-pair approximation[50]. In the limit of a full CI rotations between occupied and virtual positiveenergy orbitals become redundant. This is not the case for rotations between occupied positive-energy orbitals and virtual negative-energy orbitals which assure complete update of the projection operators in which the Dirac-Coulomb Hamiltonian is embedded.

\section{2-COMPONENT HAMILTONIANS}

\section{A. General considerations}

We now turn to a discussion of 2-component relativistic Hamiltonians. The basic idea is to generate a 2-component Hamiltonian which reproduces the positive-energy spectrum of the parent 4-component Hamiltonian. Foldy and Wouthuysen[74] proposed to look for a unitary transformation $U$ that will formally decouple the large and small components

$$
U^{\dagger}\left[\begin{array}{ll}
h_{L L} & h_{L S} \\
h_{S L} & h_{S S}
\end{array}\right] U=\left[\begin{array}{cc}
\tilde{h}_{++} & 0 \\
0 & \tilde{h}_{--}
\end{array}\right]
$$

Another approach that has been discussed in the literature is the elimination of the small component (ESC). The two approaches can be shown to be equivalent [75] and we shall focus on the latter. Let us, however, begin with the elimination of the small components. We will write the 4-component relativistic effective one-electron equation, e.g. the Dirac equation, as a system of coupled equations

$$
h_{L L} \psi^{L}+h_{L S} \psi^{S}=E \psi^{L}
$$




$$
h_{S L} \psi^{L}+h_{S S} \psi^{S}=E \psi^{S}
$$

Using the exact coupling $R$ of the large and small component (24) to eliminate $\psi^{S}$ in (35) we obtain a 2-component equation for the large component only

$$
\left[h_{L L}+h_{L S} R\right] \psi^{L}=E \psi^{L} .
$$

However, the large component is not normalized to unity and for this purpose we seek a normalization operator $N^{+}$. We set $\phi=N^{+} \psi^{L}$ and find

$$
\langle\phi \mid \phi\rangle=\langle\psi \mid \psi\rangle=\left\langle\psi^{L} \mid \psi^{L}\right\rangle+\left\langle\psi^{S} \mid \psi^{S}\right\rangle=\left\langle\psi^{L}\left|1+R^{\dagger} R\right| \psi^{L}\right\rangle
$$

from which we deduce that the normalization operator is $N_{+}=\sqrt{1+R^{\dagger} R}$. The 2-component equation of the correctly normalized function $\phi$ is accordingly

$$
h_{++} \phi=E \phi ; \quad h_{++}=N_{+}\left[h_{11}+h_{12} R\right] N_{+}^{-1}=\sqrt{1+R^{\dagger} R}\left[h_{11}+h_{12} R\right] \frac{1}{\sqrt{1+R^{\dagger} R}}
$$

An alternative route to the elimination of the small component was proposed by Dyall [76]: Summing the equations (35) and (36), the latter multiplied by $R^{\dagger}$ from the left, gives

$$
\left[h_{L L}+h_{L S} R+R^{\dagger} h_{S L}+R^{\dagger} h_{S S} R\right] \psi^{L}=E\left(1+R^{\dagger} R\right) \psi^{L} .
$$

Comparing with (38) one sees that a metric has been introduced on the right hand side of (40) which provides correct normalization of $\psi^{L}$. Equations (37) and (40) have therefore been dubbed unnormalized and normalized elimination of the small components (UESC and NESC), respectively.

Due to the explicit energy-dependence appearing in the exact coupling operator $R$ in Eq. (24) early 2-component relativistic Hamiltonians were based on approximate couplings, as will be discussed in section IIIB. However, in a very insightful paper Heully and co-workers [77] demonstrated that it is possible to derive an expression for the coupling operator $R$ without any explicit energy-dependence. Multiplying through (35) with $R$ from the left it is possible to equate the left-sides of (35) and (36)

$$
R h_{L L} \psi^{L}+R h_{L S} R \psi^{L}=E \psi^{S}=h_{S L} \psi^{L}+h_{S S} R \psi^{L}
$$

Since the large component $\psi^{L}$ is now arbitrary we arrive at an operator equation for the exact coupling $R$ without any explicit reference to energy

$$
h_{S L}+h_{S S} R=R h_{L L}+R h_{L S} R
$$

This operator equation is second order in $R$ and has accordingly two sets of solutions, $R_{+}$and $R_{-}$, corresponding to the positive- and negative-energy branch, respectively, and being of order $O\left(c^{-1}\right)$ and $O\left(c^{1}\right)$. By an analogous procedure it is also possible to arrive at an operator equation for the inverse coupling $\psi^{L}=R^{-1} \psi^{S}$ which takes the form

$$
h_{L S}+R^{-1} h_{S S}=-h_{L L} R^{-1}+R^{-1} h_{S L} R^{-1} .
$$

Taking the Hermitian conjugate of the coupling equation shows that $R^{-1}$ and $-R^{\dagger}$ fulfill the same equation. Combined with order analysis Heully et al. [77] were able to make the identifications

$$
R_{-}^{-1}=-R_{+}^{\dagger} ; \quad R_{+}^{-1}=-R_{-}^{\dagger}
$$

and to provide a general form for the Foldy-Wouthuysen transformation (34)

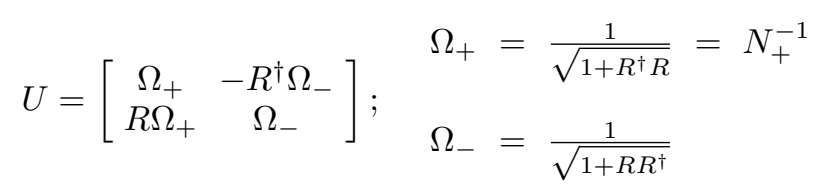

It is convenient $[28,78]$ to split the transformation into two parts $U=W_{1} W_{2}$. The first transformation

$$
W_{1}=\left[\begin{array}{cc}
1 & -R^{\dagger} \\
R & 1
\end{array}\right]
$$


decouples the large and small components whereas the second transformation

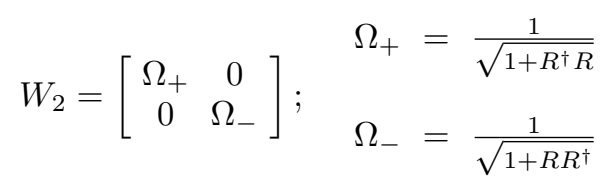

re-establishes normalization.

It is instructive to first consider the unitary decoupling transformation (45) applied to the Dirac 4-spinor

$$
U^{\dagger}\left[\begin{array}{c}
\psi^{L} \\
\psi^{S}
\end{array}\right]=\left[\begin{array}{c}
\Omega_{+}\left(\psi^{L}+R^{\dagger} \psi^{S}\right) \\
\Omega_{-}\left(\psi^{S}-R \psi^{L}\right)
\end{array}\right]
$$

For positive-energy solutions we want the lower components to be zero which leads to the relation

$$
\psi_{+}^{S}=R \psi_{+}^{L},
$$

indeed showing that $R$ can be identified with the coupling $R_{+}$between positive-energy large- and small components. Further manipulation of the positive-energy 2-component solution gives

$$
\psi_{+}^{2 c}=\frac{1}{\sqrt{1+R^{\dagger} R}}\left(\psi^{L}+R^{\dagger} \psi^{S}\right)=\frac{1}{\sqrt{1+R^{\dagger} R}}\left(\psi^{L}+R^{\dagger} R \psi^{L}\right)=\sqrt{1+R^{\dagger} R} \psi^{L},
$$

suggesting that it can be expanded in the large component basis only.

The decoupling transformation $W_{1}$ gives a Hamiltonian on the form

$$
\left[\begin{array}{cc}
h_{L L}+h_{L S} R+R^{\dagger} h_{S L}+R^{\dagger} h_{S S} R & -h_{L L} R^{\dagger}+h_{L S}-R^{\dagger} h_{S L} R^{\dagger}+R^{\dagger} h_{S S} \\
-R h_{L L}-R h_{L S} R+h_{S L}+h_{S S} R & R h_{L L} R^{\dagger}-R^{\dagger} h_{L S}-h_{S L} R^{\dagger}+h_{S S}
\end{array}\right] .
$$

It can be seen that the lower and upper off-diagonal blocks are identical to the coupling equation (42) and its Hermitian conjugate, respectively, and are accordingly zero for exact coupling $R$. The second transformation $W_{2}$ accomplishes renormalization, thus assuring unitarity of the total transformation, and generates a 2-component relativistic Hamiltonian for positive-energy solutions on the form

$$
h_{++}=\frac{1}{\sqrt{1+R^{\dagger} R}}\left[h_{11}+h_{12} R+R^{\dagger}\left(h_{21}+h_{22} R\right)\right] \frac{1}{\sqrt{1+R^{\dagger} R}} .
$$

In the case of exact coupling we can use the coupling equation (42) to simplify the Hamiltonian further to

$$
h_{++}=\sqrt{1+R^{\dagger} R}\left[h_{11}+h_{12} R\right] \frac{1}{\sqrt{1+R^{\dagger} R}} .
$$

The final 2-component equation now reads

$$
h_{++} \psi_{+}^{2 c}=E \psi \cdot_{+}^{2 c}
$$

The 2-component Hamiltonian (53) is strictly identical to the one obtained by UESC (39) and shows the equivalence of the two decoupling approaches, but only for exact coupling. On the other hand, using (52) and (50) the 2-component equation (54) is straightforwardly re-arranged to the NESC equation (40), showing their equivalence also in the case of approximate coupling. NESC can indeed be shown to provide more accurate energies in the approximate coupling case and is as such to be preferred over UESC [76, 79].

\section{B. Approximative 2-component relativistic Hamiltonians}

A first generation of 2-component relativistic Hamiltonians were developed based on approximations to the exact energy-dependent coupling (24) of the large and small components and have found their way into various widely distributed computer codes. In this section I give a brief overview of the most important Hamiltonians of this kind. The section is intentionally brief since our main focus is on exact 2-component relativistic Hamiltonians and since the approximate variants are amply described elsewhere [10, 11]. 
The Pauli Hamiltonian

$$
\hat{h}^{\text {Pauli }}(i)=V+T_{i} \underbrace{-\frac{p_{i}^{4}}{8 m^{3} c^{2}}}_{\text {mass-velocity }} \underbrace{+\frac{1}{4 m^{2} c^{2}} \boldsymbol{\sigma} \cdot\left[(\boldsymbol{\nabla} V) \times \mathbf{p}_{i}\right]}_{\text {spin-orbit }} \underbrace{+\frac{1}{8 m^{2} c^{2}}\left(\nabla^{2} V\right)}_{\text {Darwin }}
$$

is based on the approximate coupling

$$
R=\frac{1}{2 m c}\left[1+\frac{E^{+}-V}{2 m c^{2}}\right]^{-1}(\boldsymbol{\sigma} \cdot \mathbf{p}) \sim \frac{1}{2 m c}(\boldsymbol{\sigma} \cdot \mathbf{p})
$$

and is obtained by applying the unitary decoupling transformation (45) and retaining terms only to $O\left(c^{-2}\right)$, which adds three relativistic correction terms to the non-relativistic one-electron Hamiltonian. The mass-velocity term is easily recognized as a first-order correction to the kinetic energy by comparing with the relativistic free-particle energy (5). The second term is the generic form of the one-electron spin-orbit operator and is seen to not contain the orbital angular momentum operator. Upon insertion of the electrostatic potential of clamped point nuclei we obtain the more familiar form

$$
V=-\sum \frac{Z_{A} e^{2}}{r_{i A}} \quad \rightarrow \quad h^{S O}=\sum_{A} \frac{Z e^{2}}{4 m^{2} c^{2} r_{i A}^{3}} \boldsymbol{\sigma} \cdot \mathbf{l}_{A i}
$$

However, it should be noted that the orbital angular momentum operators $\mathbf{l}_{A i}$ are defined with respect to each nucleus $A$ and represents the relative motion of electron and nuclei.

The third term, the Darwin term, represents a correction to the electron-nucleus interaction,

$$
V=-e \phi(\mathbf{r}) \quad \rightarrow \quad-e \phi(\mathbf{r}+\boldsymbol{\delta}),
$$

due to Zitterbewegung. The Darwin term may be generated by Taylor-expanding the modified interaction to second order in the Zitterbewegung amplitude $\delta$ and carrying out suitable time averaging (indicated by brackets $\left.\langle\ldots\rangle_{T}\right)[27]$

$$
V+h^{\text {Darwin }}=-e \phi(\mathbf{r})-e\langle(\boldsymbol{\delta} \cdot \nabla)\rangle_{T} \phi(\mathbf{r})-\frac{1}{2} e\left\langle(\boldsymbol{\delta} \cdot \nabla)^{2}\right\rangle_{T} \phi(\mathbf{r})=-e \phi(\mathbf{r})-e \frac{\left\langle\delta^{2}\right\rangle_{T}}{6} \nabla^{2} \phi(\mathbf{r})
$$

Assuming isotropicity of the Zitterbewegung the first-order term averages to zero and only diagonal terms $\left\langle\delta_{x}^{2}\right\rangle_{T}=$ $\left\langle\delta_{x}^{2}\right\rangle_{T}=\left\langle\delta_{x}^{2}\right\rangle_{T}=\frac{1}{3}\left\langle\delta^{2}\right\rangle_{T}$ are retained in the second-order term. The precise form of the Darwin term is then obtained through the identification $\delta_{x}=\delta_{y}=\delta_{z}=\frac{1}{2 m c}$. Interestingly, this amplitude can be obtained from a hand-waving model of Zitterbewegung depicted in figure 5: In the vicinity of an electron its field is sufficiently strong to allow the creation of a electron-positron pair. The positron annihilates the original electron and the "new" electron takes over. The creation of an electron-positron pair requires an energy of at least $2 m c^{2}$. Using the energy-time uncertainty relation we deduce that the maximum particle displacement is

$$
\Delta E \Delta t \geq 1 \rightarrow \Delta x=c \Delta t \approx \frac{1}{2 m c}
$$

which corresponds exactly to the Zitterbewegung amplitude given above. This simple model accordingly suggests that the Dirac equation does not describe a single electron, rather a relay of electrons !

A nice feature of the Pauli Hamiltonian is that its underlying physics is reasonably transparent, yet it has limited applicability in relativistic molecular electronic structure calculations. One disadvantage is that the mass-velocity term has no lower bound, in contrast to the square-root expression of the relativistic free-particle energy (5) from which it is extracted. This feature bars the use of the Pauli Hamiltonian in variational calculations. The Pauli Hamiltonian has on the other hand been successfully employed to provide first-order relativistic corrections in high-precision work on interaction potentials of light atoms such as helium (see for instance [80]). A second disadvantage of the Pauli Hamiltonian is that the Darwin term and higher corrections contain derivatives of the nuclear potential and are thus highly singular terms which are difficult to describe in a finite basis approximation. $(24)$

$$
R=\frac{c}{2 m c^{2}-V}\left[1+\frac{E^{+}}{2 m c^{2}-V}\right]^{-1}(\boldsymbol{\sigma} \cdot \mathbf{p}) \sim \frac{c}{2 m c^{2}-V}(\boldsymbol{\sigma} \cdot \mathbf{p})
$$




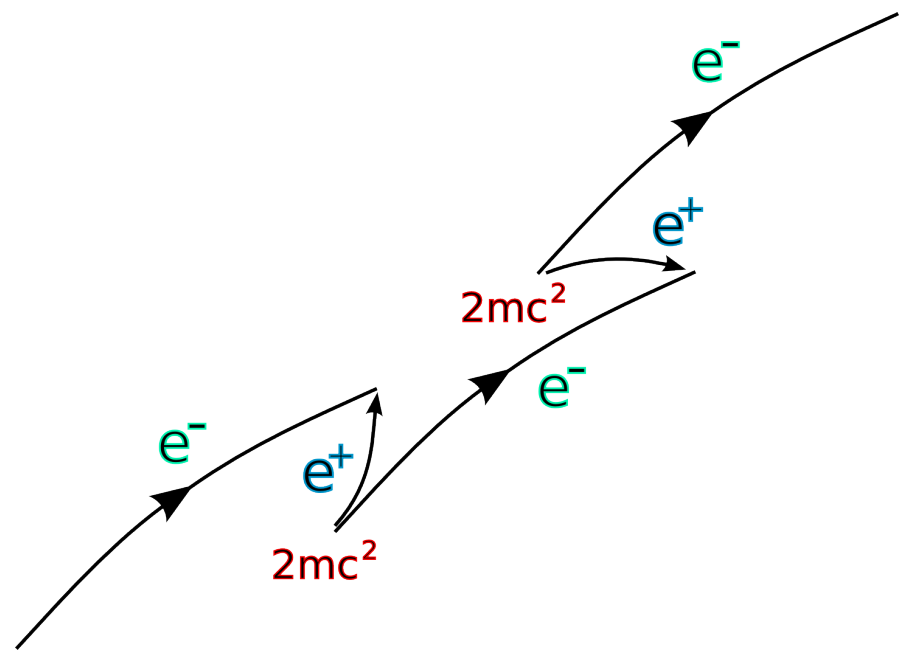

Figure 5. A pictorial model of Zitterbewegung.

Carrying out the decoupling transformation $W_{1}(46)$ of the Dirac Hamiltonian leads to the Zeroth-Order Regular Approximation (ZORA) [81-83], whereas subsequent renormalization $W_{2}(47)$ gives the infinite-order (IORA) variant [84]. The variationally stable ZORA Hamiltonian

$$
\hat{H}^{\mathrm{ZORA}}=V+T^{Z O R A} ; \quad T^{Z O R A}=\frac{1}{2 m}(\boldsymbol{\sigma} \cdot \mathbf{p}) K(\boldsymbol{r})(\boldsymbol{\sigma} \cdot \mathbf{p}) ; \quad K(\boldsymbol{r})=\frac{2 m c^{2}}{2 m c^{2}-V}
$$

contains an effective kinetic energy operator $T^{Z O R A}$ which goes into the non-relativistic one when $V \rightarrow 0$, in practice away from nuclei. As depicted in figure 6, for the mercury atom the relativistic factor $K(\boldsymbol{r})$ is essentially unity at a distance of $0.2 a_{0}$, just outside the radial expectation value of the $3 s_{1 / 2}$ orbital. Expanding $K(\boldsymbol{r})$ in orders of the potential and comparing with the Pauli Hamiltonian shows that the ZORA Hamiltonian contains no mass-velocity term, only parts of the Darwin term and the full one-electron spin-orbit term [10]. Spin-dependent and -independent parts of the effective kinetic energy operator $T^{Z O R A}$ can be separated using the Dirac identity (6). A nice feature of the ZORA Hamiltonian is that the insertion of the electronic mean-field or the full Kohn-Sham potential in $T^{Z O R A}$ automatically provides the inclusion of two-electron spin-orbit contributions. The potential term in $T^{Z O R A}$ leads on the other hand to electrical gauge-dependence in that adding a constant $\Delta$ to the potential, $V \rightarrow V+\Delta$, does not lead to the addition of the same constant to the energy, $E \rightarrow E+\Delta$, as is observed in both the Schrödinger and the Dirac equation. This gauge-dependence, which shows up in the calculation of molecular or electronic binding energies, can be curbed by using the so-called scaled ZORA equation [83], based on approximate renormalization, or by replacing the potential term $V$ by a model potential constructed from a superposition of atomic potentials[85] or densities[86].

The direct analytical calculation of $T^{Z O R A}$ in a finite basis is rather forbidding due to the presence of a potential term $V$ in the denominator and is better handled by $3 \mathrm{D}$ numerical integration, which favors implementation in the framework of density functional theory (DFT) rather than wave function theory (WFT). The first implementation of ZORA in WFT was nevertheless reported by Faas and co-workers[87-89]. A fully analytic implementation of the ZORA Hamiltonian may be obtained by setting the $S S$ block of the metric of the modified Dirac equation (29) to zero[55]. An unnormalized elimination of the small components then leads to an effective kinetic energy operator on the form

$$
T^{Z O R A}=T\left(T-W_{0}\right)^{-1} T,
$$

to be constructed in matrix representations of $T$ and $W_{0}$. Further manipulation leads to the form

$$
T^{Z O R A}=T\left(T-W_{0}\right)^{-1}\left(T-W_{0}+W_{0}\right)=T+W ; \quad W^{-1}=W_{0}^{-1}-T^{-1}
$$

employed in the analytic implementation of Filatov and Cremer [90,91] to which later a gauge-correction was added[92].

A third class of 2-component approximate relativistic Hamiltonians have been motivated by the observation that the variational instability of the Pauli Hamiltonian arises from the truncated expansion of the relativistic free-particle energy (5), whereas the complete square-root expression has a lower bound. The idea is therefore to keep the squareroot form for the kinetic energy in the final 2-component relativistic Hamiltonian. This is achieved by first carrying 


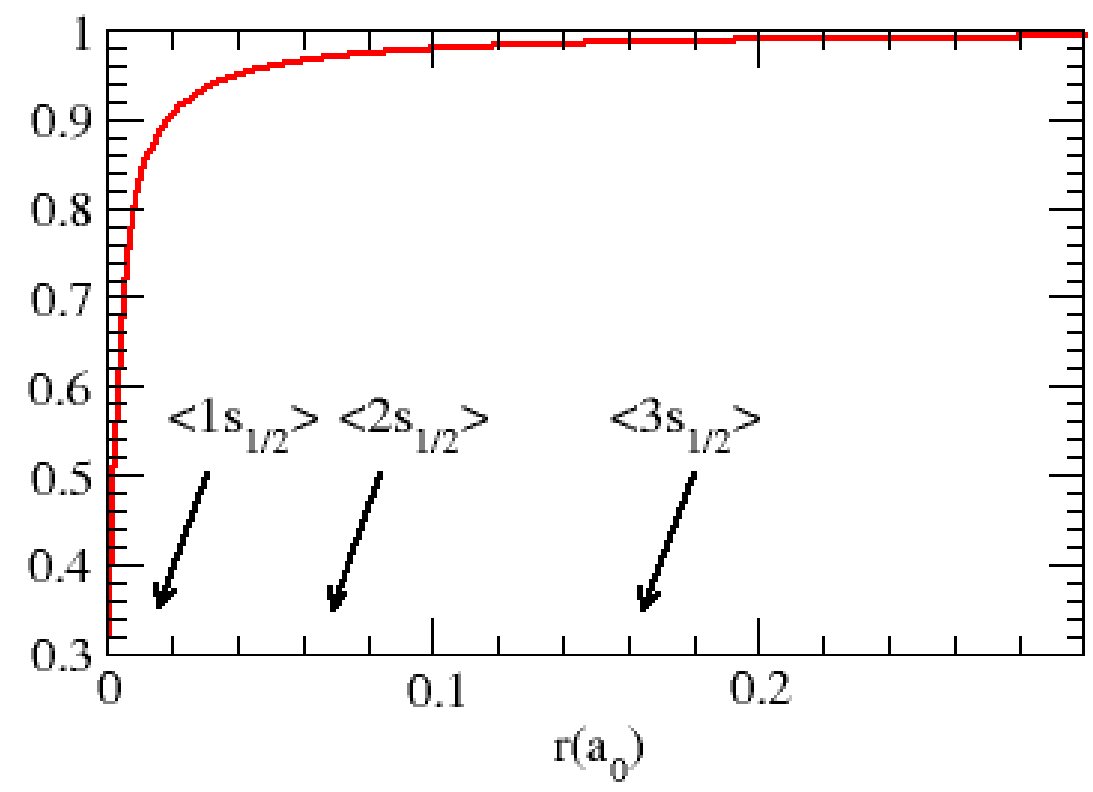

Figure 6. The factor $K(\boldsymbol{r})$ of the ZORA kinetic energy operator plotted as a function of radial distance (in $a_{0}$ ) for the mercury atom with point charge nucleus.

out a free-particle Foldy-Wouthuysen transformation $U_{0}$, which in the momentum representation has a simple closed form. Starting from the Dirac Hamiltonian in the molecular field (16) the resulting transformed Hamiltonian is given by

$$
U_{0}^{\dagger} \hat{h} U_{0}=\left[\begin{array}{cc}
E_{p}-m c^{2} & 0 \\
0 & -E_{p}-m c^{2}
\end{array}\right]+\left[\begin{array}{cc}
A\left[V+R_{0} V R_{0}\right] A & A\left[R_{0}, V\right] A \\
-A\left[R_{0}, V\right] A & A\left[V+R_{0} V R_{0}\right] A
\end{array}\right]
$$

where

$$
E_{p}=\sqrt{m^{2} c^{4}+c^{2} p^{2}} ; \quad R_{0}=\frac{c(\boldsymbol{\sigma} \cdot \mathbf{p})}{E_{p}+m c^{2}} \sim O\left(c^{-1}\right) ; \quad A \equiv Q_{0}^{+}=\sqrt{\frac{E_{p}+m c^{2}}{2 E_{p}}} \sim O\left(c^{0}\right) .
$$

The free-particle part now has the desired diagonal form and singularities arising from the potential $V$ are regulated by the kinematical factors (66), but the off-diagonal blocks of the potential part are of order unity in $V$ and $c^{-1}$, such that the total Hamiltonian $\left[U_{0}^{\dagger} \hat{h} U_{0}\right]_{++}$, denoted the first-order Douglas-Kroll-Hess (DKH1) Hamiltonian and extracted from the $(++)$ block of the transformed Hamiltonian, has only limited applicability in the relativistic domain $[93,94]$. Further decoupling is therefore necessary. Douglas and Kroll proposed decoupling through a sequence of unitary transformations

$$
U=U_{0} U_{1} U_{2} \ldots
$$

in orders of the external potential $V$ [95]. Bernd Hess devised matrix techniques for the construction of the kinematical factors (66), starting from a matrix representation of the non-relativistic kinetic energy operator in uncontracted finite standard basis sets of quantum chemistry, and thereby opened up for widespread use of this approach in quantum chemistry. His contribution can not be underestimated, and this approach is therefore justly referred to as the DouglasKroll-Hess transformation. The exact decoupling equation (24) for the free-particle transformed Hamiltonian (65) can be expressed as

$$
\left[R_{1}, E_{p}\right]_{+}=-\underbrace{A\left[R_{0}, V\right] A}_{O\left(V^{1}\right)}+\underbrace{\left[A\left[V+R_{0} V R_{0}\right] A, R_{1}\right]}_{O\left(V^{2}\right)}-\underbrace{R_{1} A\left[R_{0}, V\right] A R_{1}}_{O\left(V^{3}\right)}
$$

From order analysis in terms of the external potential the coupling is approximated as

$$
\left[R_{1}, E_{p}\right]_{+} \sim-A\left[R_{0}, V\right] A
$$


defining the approximate decoupling transformation $U_{1}$ which generates the second-order Douglas-Kroll-Hess Hamiltonian (DKH2). The original implementation by Hess stopped at this order, but the method has been extended to higher [96-98] and even arbitrary order[99, 100].

Barysz, Sadlej and Snijders carried out an order analysis of the exact coupling equation (68) in terms of $c^{-1}$ rather than the external potential [101]. The lowest-order approximation to $R_{1}$ is then

$$
R_{1} \sim-\underbrace{\frac{1}{2 m c^{2}} A[R, V] A}_{O\left(c^{-3}\right)}
$$

giving a 2-component Hamiltonian correct through order $O\left(c^{-4}\right)$, but also containing higher-order terms. Rather than performing a sequence of unitary transformations, as in the DKH-approach, they instead proposed to solve the coupling equation (68) in an iterative manner to obtain the coupling $R_{1}$ correct through some odd order $2 k-1$ in $c^{-1}$ and then perform a single unitary transformation, in addition to the free-particle one, to obtain a 2-component Hamiltonian correct through order $2 k$ in $c^{-1}$ [101-103], denoted the BSS $h_{2 k}$ Hamiltonian. The infinite-order 2component Hamiltonian generated in the limit $k \rightarrow \infty$ were given the acronym IOTC by Kedziera and Barysz [104].

\section{Exact 2-component relativistic Hamiltonians}

The development of exact 2-component relativistic Hamiltonians (X2C), reproducing exactly the positive-energy spectrum of the parent 4-component Dirac Hamiltonian, arose from the confluence of two important realizations:

1. Obtaining the exact coupling between the large and small components requires the solution, explicit or implicit, of the Dirac equation. However, the problem at hand in a typical quantum chemical calculation is to find approximate solutions to the many-electron Hamiltonian (1). The computational cost of such calculations is completely dominated by quantities associated with the two-electron term. Initially solving the one-electron problem is therefore meaningful and inexpensive.

2. The analytic expression of the exact coupling is not available in closed form. However, starting from a matrix representation of the parent 4-component Hamiltonian the entire decoupling transformation can be carried out using matrix algebra, without ever having to program integrals over additional complicated operator expressions.

The simplest algorithm for the generation of an exact 2-component relativistic Hamiltonian can then be stated in words:

1. Solve the parent 4-component one-electron equation on matrix form.

2. Extract the coupling $R(24)$ from the solutions.

3. Construct the transformation matrix $U(45)$, next $h^{X 2 C}$.

The two key realizations leading to the simple construction of the X2C Hamiltonian matured over a number of years in the relativistic quantum chemistry community, but both are present in a 1997 paper by Dyall [76]. Dyall sought to interface relativistic and non-relativistic methods for molecular electronic structure calculations within the finite basis approximation. For this purpose he devised the NESC equation (40) which goes smoothly into the Schrödinger equation in the non-relativistic limit. He does not reference the work of Heully et al. [77] and works with an explicitly energy-dependent matrix expression for the coupling $R$ ( $\boldsymbol{U}$ in his notation). He does state, though: "in principle, a single matrix $\boldsymbol{U}$ can be defined which simultaneously connects all the large and small components for the entire set of solutions, and need not to be given for each solution"[76]. As a numerical example he considers a one-electron system, $N e^{+9}$, for which he obtains NESC solutions using the energy-dependent expression of $\boldsymbol{U}$, but also extracting the coupling matrix directly from the corresponding 4-component eigenvectors.

The first computer implementation that actually delivered the X2C Hamiltonian on matrix form for use in general molecular applications was reported by Hans Jørgen Aagaard Jensen and Miroslav Iliaš and employed in a relativistic Coupled Cluster study of lead oxide [105]. The X2C Hamiltonian was constructed in the spirit of the BSS Hamiltonian, but in a simpler manner. In a first step the free-particle Dirac equation was solved on matrix form. The matrix representation of the Dirac Hamiltonian in the molecular field (16) was then transformed using the free-particle eigenvectors, a procedure which is equivalent to the free-particle Foldy-Wouthusen transformation (65). In a second step, the Dirac equation, now in free-particle basis, was solved on matrix form, the coupling extracted and a matrix representation of $h^{X 2 C}$ constructed. The implementation and application of this algorithm was presented by Jensen 
at the 2005 Conference on Relativistic Effects in Heavy Elements (REHE), but unfortunately not followed up by any publication.

The initial free-particle transformation allowed Jensen and Iliaš to construct various finite-order 2-component Hamiltonians such as the second-order DKH Hamiltonian. However, for the construction of the infinite-order 2component Hamiltonian this initial step is not needed. Kutzelnigg and Liu[54, 106, 107] therefore proposed a one-step procedure, at the time denoted exact quasirelativistic theory (XQR), for the generation of the exact 2-component oneelectron Hamiltonian, starting from a matrix representation of the modified Dirac Hamiltonian (29), and obtaining the coupling either by diagonalisation or by iterative solution of the coupling equation (42). The scheme was further developed and extended to the Kohn-Sham framework by Liu and Peng[108, 109]. A one-step procedure based on diagonalisation of the 4-component parent matrix equation was independently developed by Iliaš and Saue under the acronym IOTC [75]. To avoid a profusion of acronyms for the same object, albeit obtained by somewhat different algorithms, it was agreed[110] to use the generic acronym X2C for the eXact 2-Component Hamiltonian obtained by matrix algebra.

Let us briefly go through the one-step algorithm for the construction of the $\mathrm{X} 2 \mathrm{C}$ Hamiltonian as implemented in the DIRAC program package [9]:

1. We start from a matrix representation of the parent 4-component one-electron equation, e.g. the Dirac equation, in a finite non-orthogonal atomic orbital (AO) basis

$$
h_{0} c_{0}=S_{0} c_{0} \varepsilon .
$$

The four components of the Dirac 4-spinor are individually expanded in Cartesian Gaussian basis functions, the large and small component bases being related by unrestricted kinetic balance. The equation is subsequently transformed to orthonormal basis, using canonical orthonormalisation [111] which provides control of linear dependencies

$$
h_{1} c_{1}=c_{1} \varepsilon ; \quad h_{1}=V_{1}^{\dagger} h_{0} V_{1} ; \quad V_{1}^{\dagger} S_{0} V_{1}=I
$$

2. A matrix representation $W$ of the transformation (28) to the modified Dirac equation is constructed in the orthonormal basis and subsequently applied

$$
h_{2} c_{2}=S_{2} c_{2} \varepsilon ; \quad S_{2}=W^{\dagger} W
$$

thus enforcing restricted kinetic balance [55]. Orthonormality is restored by a transformation $V_{2}$

$$
h_{3} c_{3}=c_{3} \varepsilon ; \quad V_{2}^{\dagger} S_{2} V_{2}=I
$$

thus avoiding pitfalls [109] of carrying out the decoupling transformation $U$ in a non-orthogonal basis. The total transformation $W V_{2}$ is accordingly unitary.

3. The eigenvalue problem (73) is solved and the coupling $R$ extracted. In the matrix representation the coupling relations are given as

$$
C_{+}^{S}=R C_{+}^{L} ; \quad C_{-}^{L}=-R^{\dagger} C_{-}^{S}
$$

where $C_{+(-)}^{L(S)}$ is the block of the eigenvector matrix corresponding to the large (small) components of the positive(negative)-energy solutions. Rather than solving either equation directly we follow the original implementation of Jensen and Iliaš and construct the equation

$$
A R=B ; \quad A=C_{-}^{S} C_{-}^{S \dagger} ; \quad B=-C_{-}^{S} C_{-}^{L \dagger},
$$

where the matrix $A$ is Hermitian and positive-definite such that the system can be solved by a Cholesky decomposition [75].

4. From the coupling $R$ the decoupling transformation matrix $U$ is constructed. Note that the renormalization transformation $W_{2}(47)$ can be constructed using canonical orthonormalisation. The matrix representation of $h^{X 2 C}$ is constructed and subsequently transformed back to the initial AO-basis.

It should be noted that spin-orbit interaction can be eliminated from the 2-component Hamiltonian in two different ways, either from the parent 4-component modified Dirac Hamiltonian, or from the final 2-component Hamiltonian. The first route gives an exact 2-component Hamiltonian which reproduces exactly the positive-energy solutions of the 4-component spin-free Hamiltonian, whereas the latter route corresponds to the approach taken to eliminate spin-dependent terms of approximate Hamiltonians such as DKH2.

For large systems the diagonalisation of the Dirac Hamiltonian for the full molecule may become a bottleneck, which can be avoided by extracting the coupling at the level of individual atoms or fragments, as already proposed by Liu and co-workers $[108,112]$. 


\section{Beyond one-electron Hamiltonians}

So far our discussion of 2-component relativistic Hamiltonians has focused rather exclusively on one-electron Hamiltonians. However, construction of the full electronic Hamiltonian (1) also requires finding a suitable form for the two-electron operator at the 2-component level. The most consistent choice would be to transform the 4-component two-electron operator using the same decoupling transformation as for the one-electron part. On matrix form this can be expressed as [113]

$$
[U(1) \otimes U(2)]^{\dagger} G(1,2)[U(1) \otimes U(2)]=\left[\begin{array}{cccc}
\tilde{G}_{++}^{++} & \tilde{G}_{+-}^{+-} & \tilde{G}_{++}^{-+} & \tilde{G}_{++}^{--} \\
\tilde{G}_{++}^{++} & \tilde{G}_{+-}^{+-} & \tilde{G}_{++}^{-+} & \tilde{G}_{+-}^{-+} \\
\tilde{G}_{-+}^{++} & \tilde{G}_{-+}^{+-} & \tilde{G}_{-+}^{-+} & \tilde{G}_{--}^{--} \\
\tilde{G}_{--}^{++} & \tilde{G}_{--}^{+-} & \tilde{G}_{--}^{-+} & \tilde{G}_{--}^{--}
\end{array}\right],
$$

where $G(1,2)$ represents the supermatrix of two-electron integrals at the 4-component level. After transformation the $\tilde{G}_{++}^{++}$block would be combined with the $\tilde{h}_{++}$block from the corresponding transformation (34) of the one-electron Hamiltonian. However, the transformation involves the full set of two-electron integrals at the 4-component level and the generation of the 2-component two-electron integrals will be more expensive than the corresponding 4-component calculation. Such a scheme has nevertheless been reported by Seino and Hada [114] at the infinite-order DKH level, and reductions of computational cost are presently being sought by exploiting locality [115]. A third-order DKH scheme including the mean-field potential in the decoupling procedure was previously reported by Nakajima and Hirao [116, 117].

A straightforward approximation is to combine the 2-component one-electron Hamiltonian with the untransformed two-electron Coulomb operator. However, this translates into a neglect of two-electron spin-spin and spin-orbit contributions, the latter mandatory for a correct description of the fine structure of molecular spectra and for magnetic properties such as the g tensor. Approximate corrections to the untransformed Coulomb operator are most straightforwardly done in a DFT framework since they enter through an effective one-electron potential [86, 112, 118-120]. In a WFT framework two-electron spin-orbit corrections may be introduced by an atomic mean-field approach [121]. An example is the widely used AMFI code [122] in which the two-electron spin-orbit contributions at the first-order DKH level are constructed in a mean-field fashion for each constituent atom of the molecule and then added to the matrix representation of the molecular one-electron Hamiltonian. At the correlated CI or CC level corrections to the untransformed two-electron operator can be introduced by a molecular mean-field approach [113]. To see how this works, we re-write the second-quantized electronic Hamiltonian (33) on normal-ordered form

$$
\hat{H}=\sum_{p q} F_{p q}\left\{a_{p}^{\dagger} a_{q}\right\}+\frac{1}{2} \sum_{p q r s} g_{p q r s}\left\{a_{p}^{\dagger} a_{r}^{\dagger} a_{s} a_{q}\right\}
$$

where now appears elements $F_{p q}$ of the Fock matrix. An initial SCF calculation is carried out at the 4-component level and the decoupling transformation is then defined and carried out with respect to the converged Fock matrix. This can be justified from the higher computational scaling of the correlated calculation. The 2-component Fock matrix, which now carries infinite-order molecular mean-field spin-orbit corrections is then combined with the untransformed two-electron operator and employed at the correlated level.

An important observation is that errors introduced by an approximate treatment of the two-electron interaction at the 2-component level can be assessed by 4-component calculations [113]. Going back to the unitary decoupling transformation (34) it is evident that an Hamiltonian which reproduces the positive-energy solutions only of the parent 4-component Hamiltonian is simply obtained by full diagonalisation of the parent 4-component Hamiltonian and then backtransformation using only the positive-energy solutions. This is not a very useful approach in the sense that the solutions must still be expanded in bases for both the large and small components, but it tells us that the generation of the 2-component exact Hamiltonian $\tilde{h}_{++}$is equivalent to projecting out the negative-energy solutions of the parent 4 component Hamiltonian. Applying the same projectors to the two-electron part in 4-component calculations therefore mimics 2-component calculations with the correctly transformed two-electron operator. The above observation also tells us that a atom- or fragment-based construction of the X2C Hamiltonian should preferably start from converged Fock (Kohn-Sham) matrix to allow maximally relaxed projectors.

\section{E. Picture change errors}

We have seen in section III A that 2-component Hamiltonians are obtained by taking the ++ block of the transformed 4-component parent Hamiltonian, that is

$$
h^{2 c}=\left[U^{\dagger} h^{4 c} U\right]_{++} .
$$


For consistency 4-component property operators $\Omega^{4 c}$ must be subjected to the same decoupling transformation as the Hamiltonian, that is

$$
\Omega^{2 c}=\left[U^{\dagger} \Omega^{4 c} U\right]_{++}
$$

Use of the approximate expression

$$
\Omega^{2 c} \approx\left[\Omega^{4 c}\right]_{L L}
$$

leads to picture change errors that may be larger than the relativistic effects [123-126]. A nice feature of the simple algorithm for the construction of the $\mathrm{X} 2 \mathrm{C}$ Hamiltonian is that it provides an explicit representation of the transformation matrix $U$ such that property operators can be transformed on the fly, thus avoiding picture change errors, as well as the programming of new integrals.

As an example we may consider the electron density in some point $\boldsymbol{P}$ which is formally to be calculated as the expectation value of the corresponding operator. However, at the 4-component SCF level the expectation value reduces to the familiar sum of products of the occupied orbitals

$$
\rho^{4 c}(\boldsymbol{P})=-e \sum_{i}\left\langle\psi_{i}^{4 c}|\delta(\boldsymbol{r}-\boldsymbol{P})| \psi_{i}^{4 c}\right\rangle=-e \sum_{i} \psi_{i}^{4 c \dagger}(\boldsymbol{P}) \psi_{i}^{4 c}(\boldsymbol{P}) .
$$

This is not the case at the 2-component level where the picture change transformation leads to a modification (smearing) of the Dirac delta function

$$
\rho^{2 c}(\boldsymbol{P})=-e \sum_{i}\left\langle\psi_{i}^{2 c}\left|\left[U^{\dagger} \delta(\boldsymbol{r}-\boldsymbol{P}) U\right]_{++}\right| \psi_{i}^{2 c}\right\rangle \neq-e \sum_{i} \psi_{i}^{2 c \dagger}(\boldsymbol{P}) \psi_{i}^{2 c}(\boldsymbol{P}) .
$$

The difference is illustrated in figure 7 where we trace the electron number density of the mercury atom, calculated as orbital products, as a function of radial distance at the 4- and 2-component level. Close to the origin the two curves deviate significantly. The difference is appreciable, though, only within the radial expectation value $\langle r\rangle_{1 s}$ of the $1 s$ orbital and basically disappears on the more chemical scale employed in figure 8 , and so one may question its chemical relevance. There are, however, a number of molecular properties which probe the electron density near nuclei, such as NMR parameters, electric field gradients, molecular gradients as well as Mössbauer isomer shifts, and which provide local information with great sensitivity to the chemical environment. For such properties proper picture change transformation is crucial in order to obtain meaningful results at the 2-component relativistic level, as for instance shown in our recent study of the Mössbauer isomer shifts of mercury fluorides [127].

\section{NUMERICAL EXAMPLE}

It is beyond the scope of the present mini-review to furnish detailed benchmark studies of the relativistic Hamiltonians described in the text. However, to provide a first impression of their performance and to illustrate various features discussed in the text I give in Table I orbital energies of the mercury atom extracted from Hartree-Fock calculations using various relativistic Hamiltonians. All results have been obtained with the DIRAC code [9] with identical basis sets, such that all differences can be unambiguously attributed to the difference between Hamiltonians. Our reference will be the results obtained with the 4-component Dirac-Coulomb (DC) Hamiltonian, but I also display results obtained with the Dirac-Coulomb-Gaunt (DCG) Hamiltonian. When adding the Gaunt operator one notes an appreciable upward shift of the orbital energies of the inner core orbitals. Spin-orbit splittings $\Delta_{S O}$ are somewhat reduced due to the inclusion of spin-other orbit interaction. As discussed in section IIC, SCF calculations based on the DC (or DCG) Hamiltonian excludes negative-energy orbitals from the construction of the mean-field potential, which corresponds to the implicit use of projection operators, as suggested by Mittleman [66]. In Table I I also report results obtained with other choices of projection operators. It can be seen that the use of projection operators $P_{\text {free }}$ constructed from solutions to the free-particle Dirac equation introduce significant errors compared to the fully relaxed DC results. The errors associated with the use of projection operators $P_{V e x t}$ constructed from solutions of the bare-nucleus Dirac Hamiltonian, which defines the Dirac equation for an electron in the molecular field (16), are on the other hand quite acceptable. 


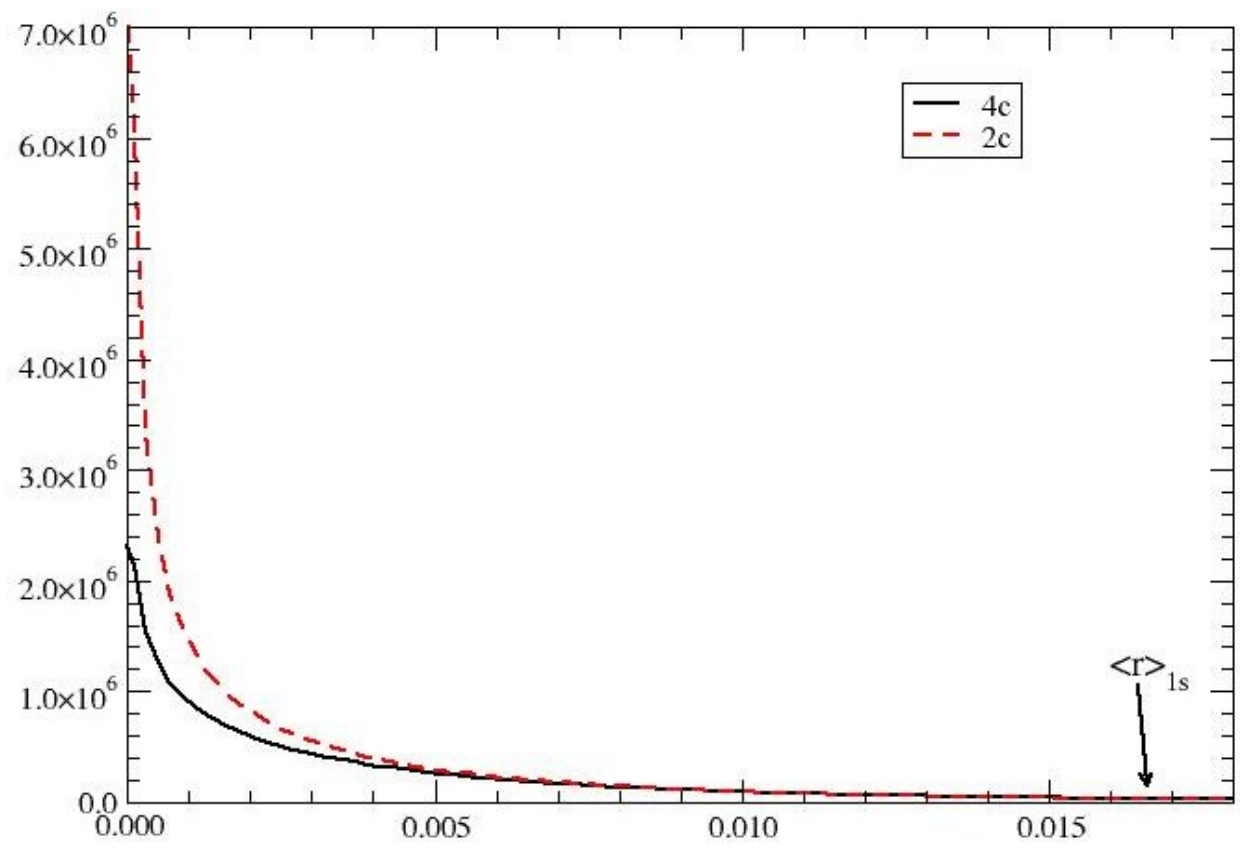

Figure 7. Comparison of 4- and 2-component electron number density, the latter calculated without picture change, of the mercury atom, as a function of radial distance. All quantities in atomic units.

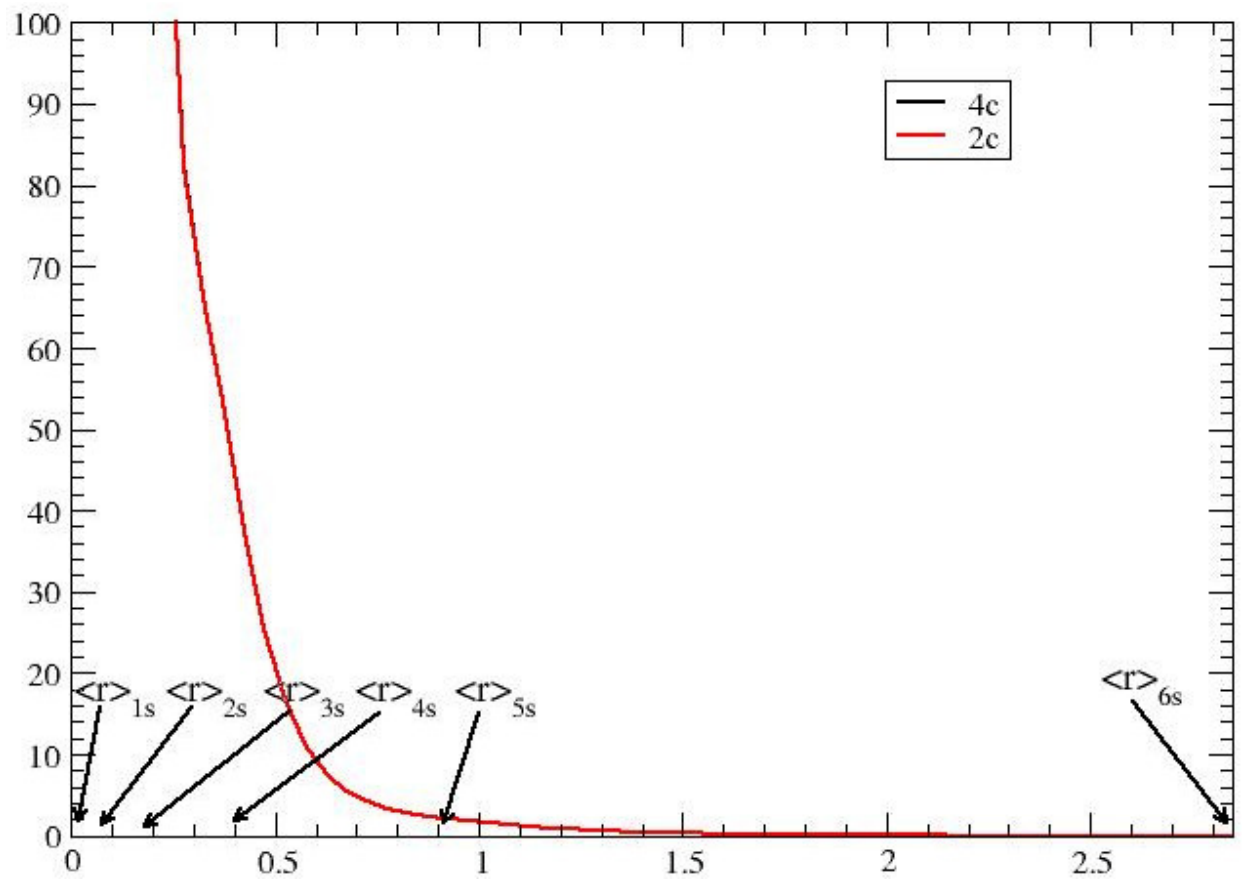

Figure 8. Comparison of 4- and 2-component electron number density, the latter calculated without picture change, of the mercury atom, as a function of radial distance. All quantities in atomic units.

Turning next to 2-component relativistic Hamiltonians we first consider the results obtained with the X2C Hamiltonian. The exact decoupling transformation (34) is based on the bare-nucleus Dirac Hamiltonian and the 2-component one-electron Hamiltonian generated in this manner reproduces to machine precision the positive-energy spectrum of the parent Hamiltonian. As two-electron operator we employ the untransformed Coulomb operator combined with two-electron spin-same orbit atomic corrections from the AMFI package [122]. As discussed in section IIID, the picture change errors introduced by not using the consistently transformed two-electron operator can be assessed by com- 
paring with the results obtained by 4-component DC calculations using bare-nucleus projectors, denoted $\mathrm{DC}\left(P_{V e x t}\right)$ in Table I. It can be seen that with the exception of the $1 s_{1 / 2}$ orbitals the deviation with respect to the reference is quite small. This also holds for the spin-orbit splittings $\Delta_{S O}$.

We next consider the results obtained with the two lowest-order Douglas-Kroll-Hess Hamiltonians. The one-electron Hamiltonians have been combined with the untransformed Coulomb term and two-electron spin-orbit corrections from the AMFI package [122]. The first-order Douglas-Kroll-Hess Hamiltonian DKH1 is obtained, as discussed in section IIIB, by a free-particle Foldy-Wouthuysen transformation. As this transformation provides an exact decoupling of the free-particle Dirac Hamiltonian, the DKH1 Hamiltonian could also be denoted X2C (free). The results obtained with the DKH1 Hamiltonian are accordingly comparable to the 4-component DC results using free-particle operators, denoted $\mathrm{DC}\left(P_{\text {free }}\right)$ in Table I, the differences being due to picture-change errors from the two-electron term. It can indeed be seen that both Hamiltonians, DKH1 and DC $\left(P_{\text {free }}\right)$, have comparable poor performance and can not be recommended for use in quantum chemical calculations. The second-order Douglas-Kroll-Hess Hamiltonian DKH2 has a significant better performance, which explains why it has found such widespread use in quantum chemistry codes.

Interestingly, the ZORA Hamiltonian has larger errors in the inner core region than the $\mathrm{DC}\left(P_{\text {free }}\right)$ Hamiltonian, but reproduces the reference DC Hamiltonian quite well for outer core and valence orbitals. The ZORA numbers in Table I have been generated using an analytic implementation based on (63). The scaled ZORA Hamiltonian, denoted scZORA in Table I, outperforms the DKH2 Hamiltonian and approaches the X2C Hamiltonian in accuracy.

\section{SUMMARY AND OUTLOOK}

None of the many-electron Hamiltonians discussed in this mini-review are truly relativistic, in the sense of being Lorentz invariant. The time-dependent Schrödinger equation is manifestly non-relativistic since it involves secondderivatives in the spatial coordinates and a first-derivative in the time coordinate. This is contrary to the theory of special theory in which time and space are treated on an equal footing to within a sign dictated by the metric. As such, the Dirac equation for an electron in a molecular field (16) also appears to violate Lorentz invariance. This equation can, however, be derived from a time-dependent Lorentz invariant Dirac equation by fixing the reference frame to the nuclear one and then factor off time by standard mathematical procedures. A fully relativistic approach to many-electron systems is on the other hand necessarily time-dependent due to the effects of retardation in the two-electron interaction, which in principle requires a complete specification of the history of the interacting particles. Relativistic Hamiltonians for chemistry are therefore chosen according to their ability to capture essential relativistic effects combined with their ease of use.

The development of exact 2-component relativistic Hamiltonians undoubtedly constitute a major breakthrough of relativistic quantum chemistry due to the simplicity of their construction and the superior accuracy with respect to approximate 2-component relativistic Hamiltonians. Removing the need for a small component basis gives a speedup of an order of magnitude at the SCF and 4-index transformation level compared to 4-component calculations, although by suitable tricks it is possible to reduce the computational cost at the latter level to that of 2-component calculations, at least in a DFT framework [112]. At the correlated CI and CC level, the cost of 2- and 4-component calculations is strictly identical, since both levels employ the no-pair approximation. This is easily seen from the second quantized form of the electronic Hamiltonian (33): It is defined in terms of one- and two-electron integrals, so once the 4-index transformation has been carried out, it is not possible to deduce whether the orbitals came from a 2- or 4-component calculation.

What is lost with respect to 4-component theory is the real-space representation of quantities such as the charge and current density, since the X2C Hamiltonian and property operators are formulated in matrix algebra. One possible option is to backtransform 2-component orbitals to the 4-component level for constructing such objects. Another complication with respect to the 4-component level is that when calculating energy derivatives for molecular properties and gradients, such derivatives have to be taken with respect to the decoupling transformation as well [130, 131]. Some consideration is also required for properties, such as NMR parameters, where the response contribution from negativeenergy orbitals can not be neglected [132-134].

With respect to approximate 2-component Hamiltonians the $\mathrm{X} 2 \mathrm{C}$ Hamiltonian further benefits from the ease of picture change transformation, making the calculation of molecular properties much more simple to implement. One may ask what will be the future for approximate Hamiltonians such as ZORA and DKH2. Both are widely distributed in computer codes and will therefore continue to be used. I personally believe that further development of the DKH Hamiltonian will eventually cease, whereas the ZORA Hamiltonian may have a brighter future due to the more transparent decoupling transformation. 
DCG DC DC $\left(P_{\text {free }}\right) \quad \mathrm{DC}\left(P_{\text {Vext }}\right) \quad \mathrm{X} 2 \mathrm{C}\left(V_{\text {ext }}\right) \quad$ DKH1 $\quad$ DKH2 $\quad$ ZORA scZORA

\begin{tabular}{|c|c|c|c|c|c|c|c|c|c|}
\hline $1 \mathrm{~s}_{1 / 2}$ & -3062.411 & -3074.239 & -3218.372 & -3074.248 & -3069.951 & -3214.349 & -3061.460 & -3380.366 & -3067.397 \\
\hline $2 \mathrm{~s}_{1 / 2}$ & -548.905 & -550.250 & -566.498 & -550.252 & -549.615 & -565.900 & -548.677 & -561.253 & -549.292 \\
\hline $2 \mathrm{p}_{1 / 2}$ & -524.536 & -526.846 & -531.525 & -526.850 & -526.451 & -531.271 & -526.045 & -537.952 & -526.461 \\
\hline $2 \mathrm{p}_{3 / 2}$ & -453.605 & -455.153 & -455.092 & -455.155 & -454.824 & -454.786 & -454.829 & -463.518 & -454.746 \\
\hline $3 \mathrm{~s}_{1 / 2}$ & -132.861 & -133.110 & -136.662 & -133.110 & -132.974 & -136.534 & -132.769 & -133.861 & -132.904 \\
\hline $3 \mathrm{p}_{1 / 2}$ & -122.195 & -122.634 & -123.707 & -122.634 & -122.557 & -123.659 & -122.463 & -123.439 & -122.564 \\
\hline $3 p_{3 / 2}$ & -106.261 & -106.541 & -106.476 & -106.541 & -106.477 & -106.416 & -106.482 & -107.145 & -106.460 \\
\hline $3 \mathrm{~d}_{3 / 2}$ & -89.242 & -89.432 & -89.228 & -89.432 & -89.418 & -89.216 & -89.430 & -89.980 & -89.418 \\
\hline $3 \mathrm{~d}_{5 / 2}$ & -85.887 & -86.016 & -85.817 & -86.016 & -85.989 & -85.788 & -86.000 & -86.505 & -85.981 \\
\hline $4 \mathrm{~s}_{1 / 2}$ & -30.593 & -30.644 & -31.527 & -30.644 & -30.613 & -31.498 & -30.562 & -30.670 & -30.596 \\
\hline $4 p_{1 / 2}$ & -26.026 & -26.119 & -26.371 & -26.120 & -26.104 & -26.363 & -26.082 & -26.168 & -26.107 \\
\hline $4 p_{3 / 2}$ & -22.131 & -22.184 & -22.153 & -22.184 & -22.172 & -22.141 & -22.174 & -22.215 & -22.169 \\
\hline $4 d_{3 / 2}$ & -14.767 & -14.792 & -14.733 & -14.792 & -14.792 & -14.733 & -14.796 & -14.821 & -14.793 \\
\hline $4 d_{5 / 2}$ & -14.036 & -14.048 & -13.990 & -14.048 & -14.045 & -13.987 & -14.049 & -14.070 & -14.044 \\
\hline $4 f_{5 / 2}$ & -4.476 & -4.469 & -4.424 & -4.469 & -4.472 & -4.428 & -4.475 & -4.480 & -4.474 \\
\hline $4 f_{7 / 2}$ & -4.321 & -4.307 & -4.264 & -4.307 & -4.310 & -4.266 & -4.313 & -4.316 & -4.310 \\
\hline $5 \mathrm{~s}_{1 / 2}$ & -5.091 & -5.099 & -5.267 & -5.099 & -5.094 & -5.262 & -5.084 & -5.093 & -5.090 \\
\hline $5 \mathrm{p}_{1 / 2}$ & -3.520 & -3.533 & -3.568 & -3.533 & -3.532 & -3.568 & -3.528 & -3.534 & -3.532 \\
\hline $5 p_{3 / 2}$ & -2.832 & -2.838 & -2.822 & -2.838 & -2.837 & -2.821 & -2.838 & -2.838 & -2.836 \\
\hline $5 d_{3 / 2}$ & -0.646 & -0.646 & -0.630 & -0.646 & -0.647 & -0.631 & -0.648 & -0.648 & -0.647 \\
\hline $5 \mathrm{~d}_{5 / 2}$ & -0.572 & -0.571 & -0.556 & -0.571 & -0.572 & -0.557 & -0.573 & -0.572 & -0.572 \\
\hline $6 s_{1 / 2}$ & -0.326 & -0.326 & -0.341 & -0.326 & -0.326 & -0.340 & -0.325 & -0.325 & -0.325 \\
\hline$\Delta_{S O}(2 \mathrm{p})$ & 70.931 & 71.693 & 76.433 & 71.695 & 71.628 & 76.485 & 71.216 & 74.434 & 71.715 \\
\hline$\Delta_{S O}(3 \mathrm{p})$ & 15.934 & 16.093 & 17.230 & 16.093 & 16.080 & 17.242 & 15.980 & 16.294 & 16.104 \\
\hline$\Delta_{S O}(4 \mathrm{p})$ & 3.895 & 3.935 & 4.218 & 3.935 & 3.932 & 4.221 & 3.908 & 3.953 & 3.939 \\
\hline$\Delta_{S O}(5 \mathrm{p})$ & 0.688 & 0.695 & 0.746 & 0.695 & 0.695 & 0.747 & 0.691 & 0.697 & 0.696 \\
\hline$\Delta_{S O}(3 \mathrm{~d})$ & 3.354 & 3.416 & 3.412 & 3.416 & 3.429 & 3.428 & 3.429 & 3.476 & 3.438 \\
\hline$\Delta_{S O}(4 \mathrm{~d})$ & 0.730 & 0.744 & 0.743 & 0.744 & 0.747 & 0.747 & 0.747 & 0.751 & 0.749 \\
\hline$\Delta_{S O}(5 \mathrm{~d})$ & 0.074 & 0.075 & 0.074 & 0.075 & 0.075 & 0.074 & 0.075 & 0.076 & 0.076 \\
\hline$\Delta_{S O}(4 \mathrm{f})$ & 0.155 & 0.161 & 0.160 & 0.161 & 0.162 & 0.162 & 0.163 & 0.164 & 0.164 \\
\hline
\end{tabular}

Table I. Orbital energies (in $E_{h}$ ) from Hartree-Fock calculations on the mercury atom based on various relativistic Hamiltonians. All calculations were carried out by the DiRAC code [9] and employed an uncontracted 24s19p12d9f large component Gaussian basis $[128,129]$ and, where relevant, a small component basis generated by restricted kinetic balance. For an explanation of acronyms, see text.

With the development of efficient 2-component methods for relativistic molecular electronic structure calculations, one may ask if relativity in chemistry has been solved, and so all that remains in this domain is the straightforward and possibly tedious transfer of the full repertoire of non-relativistic methodology to the relativistic domain. Personally I do not believe so. There are challenges ahead, notably exploring the interface with QED [29, 50], which may be of chemical relevance [? ], and the relativistic definition of certain molecular properties, such as the spin-rotation constant [135]. A major challenge is also to develop efficient electron correlation methods which incorporate spin-orbit interaction from the start [136]. Faced with bombastic statements about the completeness of relativistic molecular quantum mechanics it may be appropriate to end this mini-review with the following quote from a speech of Albert A. Michelson at the dedication of Ryerson Physics Lab, University of Chicago, in 1894 : "The more important fundamental laws and facts of the physical sciences have all been discovered and these are so firmly established that the possibility of ever being supplanted in consequence of new discoveries is exceedingly remote ... Our future discoveries must be looked for in the sixth place of decimals." I believe that nobody knows what surprises relativistic molecular quantum mechanics may have in store for us. 
[1] W. J. Hehre, L. Radom, P. v. R. Schleyer, and J. A. Pople, Ab Initio Molecular Orbital Theory (John Wiley \& Sons, New York, 1986).

[2] J. A. Pople, Rev. Mod. Phys. 71, 1267 (1999).

[3] R. Zaleśny, M. Papadopoulos, P. Mezey, and J. Leszczynski, eds., Linear-Scaling Techniques in Computational Chemistry and Physics (Springer, Dordrecht, 2011).

[4] P. Pyykkö, Chem. Rev. 88, 563 (1988).

[5] T. Saue, Adv. Quantum Chem. 48, 383 (2005).

[6] O. Fossgaard, O. Gropen, M. C. Valero, and T. Saue, J. Chem. Phys. 118, 10418 (2003).

[7] S. Wilson, J. Mol. Struct. (Theochem) 547, 279 (2001).

[8] W. Liu, Mol. Phys. 108, 1679 (2010).

[9] DIRAC, a relativistic ab initio electronic structure program, Release DIRAC10 (2010), written by T. Saue, L. Visscher and H. J. Aa. Jensen, with contributions from R. Bast, K. G. Dyall, U. Ekström, E. Eliav, T. Enevoldsen, T. Fleig, A. S. P. Gomes, J. Henriksson, M. Iliaš, Ch. R. Jacob, S. Knecht, H. S. Nataraj, P. Norman, J. Olsen, M. Pernpointner, K. Ruud, B. Schimmelpfennig, J. Sikkema, A. Thorvaldsen, J. Thyssen, S. Villaume, and S. Yamamoto (see http://dirac.chem.vu.nl).

[10] K. G. Dyall and K. Fægri, Introduction to Relativistic Quantum Chemistry (Oxford University Press, 2007).

[11] M. Reiher and A. Wolf, Relativistic Quantum Chemistry: The Fundamental Theory of Molecular Science (Wiley-VCH, 2009).

[12] P. Schwerdtfeger, ed., Relativistic Electronic Structure Theory. Part 1. Fundamentals (Elsevier, Amsterdam, 2002).

[13] P. Schwerdtfeger, ed., Relativistic Electronic Structure Theory. Part 2. Applications (Elsevier, Amsterdam, 2004).

[14] M. Barysz and Y. Ishikawa, eds., Relativistic Methods for Chemists (Springer, Dordrecht, 2010).

[15] S. Wilson and U. Kaldor, eds., Theoretical Chemistry and Physics of Heavy and Superheavy Elements (Kluwer, Dordrecht, 2003).

[16] M. Dolg and X. Cao, Chem. Rev. 112, 403 (2012).

[17] P. Schwerdtfeger, in Theoretical Chemistry and Physics of Heavy and Superheavy Elements, edited by S. Wilson and U. Kaldor (Kluwer, Dordrecht, 2003) pp. 399-438.

[18] L. Seijo and Z. Barandiarán, in Computational chemistry: Review of current trends, Vol. 4, edited by J. Leszczynski (World Scientific, Singapore, 1999) pp. 55-152.

[19] M. Klobukowski, S. Huzinaga, and Y. Sakai, in Computational chemistry: Review of current trends, Vol. 3, edited by J. Leszczynski (World Scientific, Singapore, 1999) pp. 49-74.

[20] D. H. Whiffen, Pure Appl. Chem 50, 75 (1978).

[21] P. A. M. Dirac, Proc. Roy. Soc. London A 117, 610 (1928).

[22] P. A. M. Dirac, Proc. Roy. Soc. London A 118, 351 (1928).

[23] B. L. van der Waerden, Group Theory and Quantum Mechanics (Springer, Berlin, 1974) (Translation of the German Original Edition: Die Grundlehren der mathematischen Wissenschaften Band 37, Die Gruppentheoretische Methode in der Quantenmechanik. Publisher: Verlag von Julius Springer, Berlin 1932).

[24] M. Gell-Mann, Il Nuovo Cimento 4, 848 (1956).

[25] K. Schwarzschild, Gött. Nach., Math.-Phys. Kl. , 126 (1903).

[26] W. E. Baylis and G. Jones, J. Math. Phys. 29, 57 (1988).

[27] R. E. Moss, Advanced Molecular Quantum Mechanics (Chapmann and Hall, London, 1973).

[28] W. Kutzelnigg, in Relativistic Electronic Structure Theory - Part 1. Fundamentals, edited by P. Schwerdtfeger (Elsevier, Amsterdam, 2002) p. 664.

[29] W. Kutzelnigg, Chem. Phys. 395, 16 (2011).

[30] T. Saue, in Relativistic Electronic Structure Theory. Part 1. Fundamentals, edited by P. Schwerdtfeger (Elsevier, Amsterdam, 2002) p. 332.

[31] C. G. Darwin, Phil.Mag. 39, 537 (1920).

[32] E. Schrödinger, Sitzungber. Preuss. Akad. Wiss. Physik-Math. 24, 418 (1930).

[33] G. Breit, Phys.Rev. 34, 553 (1929).

[34] K.-N. Huang, J. Chem. Phys. 71, 3830 (1979).

[35] J. A. Gaunt, Proc. Roy. Soc. London A122, 513 (1929).

[36] T. Saue, Principles and Applications of Relativistic Molecular Calculations, Ph.D. thesis, University of Oslo (1996), online version: http://dirac.chem.sdu.dk/thesis/.

[37] O. Visser, L. Visscher, P. J. C. Aerts, and W. C. Nieuwpoort, Theor. Chim. Acta 81, 405 (1992).

[38] Y.-K. Kim, Phys.Rev. 154, 17 (1967), erratum: ibid. 159, 190, (1967).

[39] F. Rosicky and F. Mark, Theor. Chim. Acta 54, 35 (1979).

[40] F. Mark, H. Lischka, and F. Rosicky, Chem. Phys. Lett. 71, 507 (1980).

[41] I. P. Grant, Phys. Rev. A 25, 1230 (1982).

[42] A. D. McLean and Y. S. Lee, in Current Aspects of Quantum Chemistry 1981, Vol. 21, edited by R. Carbo (Elsevier, Amsterdam, 1982) pp. 219-238.

[43] Y. S. Lee and A. D. McLean, J. Chem. Phys. 76, 735 (1982).

[44] K. G. Dyall, I. P. Grant, and S. Wilson, J. Phys. B 17, 493 (1984). 
[45] Y. Ishikawa and H. M. Quiney, Int. J. Quant. Chem.: Quant. Chem. Symp. 21, 523 (1987).

[46] O. Visser, P. J. C. Aerts, D. Hegarty, and W. C. Nieuwpoort, Chem. Phys. Lett. 134, 34 (1987).

[47] L. Visscher and K. G. Dyall, At. Data Nucl. Data Tables 67, 207 (1997).

[48] R. E. Stanton and S. Havriliak, J. Chem. Phys. 81, 1910 (1984).

[49] L. Visscher, P. J. C. Aerts, O. Visser, and W. C. Nieuwpoort, Int. J. Quant. Chem.: Quant. Chem. Symp. 25, 131 (1991).

[50] T. Saue and L. Visscher, in Theoretical Chemistry and Physics of Heavy and Superheavy Elements, edited by S. Wilson and U. Kaldor (Kluwer, Dordrecht, 2003) p. 211.

[51] K. G. Dyall, I. P. Grant, C. T. Johnson, F. A. Parpia, and E. P. Plummer, Comput. Phys. Comm. 55, 425 (1989).

[52] W. Kutzelnigg, Int. J Quant. Chem. 25, 107 (1984).

[53] K. G. Dyall, J. Chem. Phys. 100, 2118 (1994).

[54] W. Kutzelnigg and W. Liu, J. Chem. Phys. 123, 241102 (2005).

[55] L. Visscher and T. Saue, J. Chem. Phys. 113, 3996 (2000).

[56] L. Visscher and E. van Lenthe, Chem. Phys. Lett. 306, 357 (1999).

[57] J.-M. Lévy-Leblond, Commun. Math. Phys. 6, 286 (1967).

[58] A. Rutkowski, J. Phys. B: At. Mol. Opt. Phys. 19, 149 (1986).

[59] W. Kutzelnigg, Z.Phys.D 11, 15 (1989).

[60] W. Kutzelnigg, Z.Phys.D 15, 27 (1990).

[61] S. Stopkowicz and J. Gauss, The Journal of Chemical Physics 134, 064114 (2011).

[62] S. Stopkowicz and J. Gauss, J. Chem. Phys. 134, 204106 (2011).

[63] W. Schwalbach, S. Stopkowicz, L. Cheng, and J. Gauss, The Journal of Chemical Physics 135, 194114 (2011).

[64] G. E. Brown and D. G. Ravenhall, Proc. Roy. Soc. London A208, 552 (1951).

[65] J. Sucher, Phys. Rev. A 22, 348 (1980).

[66] M. H. Mittleman, Phys.Rev.A 24, 1167 (1981).

[67] J. D. Talman, Phys. Rev. Lett. 57, 1091 (1986).

[68] L. LaJohn and J. D. Talman, Chem. Phys. Lett. 189, 383 (1992).

[69] J.-L. Heully, I. Lindgren, E. Lindroth, and A.-M. Mårtensson-Pendrill, Phys.Rev. A 33, 4426 (1986).

[70] C. F. Bunge, R. Jauregui, and E. Ley-Koo, Int. J Quant. Chem. 70, 805 (1998).

[71] Y. Watanabe, H. Nakano, and H. Tatewaki, J. Chem. Phys. 126, 174105 (2007).

[72] Y. Watanabe, H. Nakano, and H. Tatewaki, J. Chem. Phys. 132, 124105 (2010).

[73] G. Pestka, M. Bylicki, and J. Karwowski, J. Phys. B: At. Mol. Opt. Phys. 39, 2979 (2006).

[74] L. L. Foldy and S. A. Wouthuysen, Phys.Rev. 78, 29 (1950).

[75] M. Iliaš and T. Saue, J. Chem. Phys. 126, 064102 (2007).

[76] K. Dyall, J. Chem. Phys. 106, 9618 (1997).

[77] J.-L. Heully, I. Lindgren, E. Lindroth, S. Lundqvist, and A.-M. Mårtensson-Pendrill, J. Phys. B: At. Mol. Opt. Phys. 19, 2799 (1986).

[78] W. Kutzelnigg, J. Chem. Phys. 110, 8283 (1999).

[79] K. Dyall, in Theoretical Chemistry and Physics of Heavy and Superheavy Elements, edited by S. Wilson and U. Kaldor (Kluwer, Dordrecht, 2003) p. 325.

[80] M. Przybytek, W. Cencek, J. Komasa, G. Łach, B. Jeziorski, and K. Szalewicz, Phys. Rev. Lett. 104, 183003 (2010).

[81] C. Chang, M. Pelissier, and P. Durand, Phys.Scr. 34, 394 (1986).

[82] E. van Lenthe, E. J. Baerends, and J. G. Snijders, J. Chem. Phys. 99, 4597 (1993).

[83] E. van Lenthe, E. J. Baerends, and J. G. Snijders, J. Chem. Phys. 101, 9783 (1994).

[84] K. G. Dyall and E. van Lenthe, J. Chem. Phys. 111, 1366 (1999).

[85] P. H. T. Philipsen, E. van Lenthe, J. G. Snijders, and E. J. Baerends, Phys. Rev. B 56, 13556 (1997).

[86] C. van Wüllen, J. Chem. Phys. 109, 392 (1998).

[87] S. Faas, J. G. Snijders, J. H. van Lenthe, and E. J. Baerends, Chem. Phys. Lett. 246,632 (1995).

[88] S. Faas, J. G. Snijders, and J. H. van Lenthe, in Quantum Systems in Chemistry and Physics, Vol. 1, edited by A. Hernández-Laguna, J. Maruani, R. McWeeny, and S. Wilson (Kluwer, Dordrecht, 2000) p. 251, Available at http://theochem.chem.rug.nl/publications/.

[89] S. Faas, J. H. van Lenthe, and J. G. Snijders, Mol. Phys. 98, 1467 (2000).

[90] M. Filatov, Chem. Phys. Lett. 365, 222 (2002).

[91] M. Filatov and D. Cremer, J. Chem. Phys. 118, 6741 (2003).

[92] M. Filatov and D. Cremer, J. Chem. Phys. 122, 044104 (2005).

[93] R. Buenker, P. Chandra, and B. Hess, Chem. Phys. 84, 1 (1984).

[94] J. Almlof, K. Faegri, and H. Grelland, Chem. Phys. Lett. 114, 53 (1985).

[95] M. Douglas and N. M. Kroll, Ann.Phys. 82, 89 (1974).

[96] T. Nakajima and K. Hirao, J. Chem. Phys. 113, 7786 (2000).

[97] A. Wolf, M. Reiher, and B. A. Hess, J. Chem. Phys. 117, 9215 (2002).

[98] C. van Wüllen, J. Chem. Phys. 120, 7307 (2004).

[99] M. Reiher and A. Wolf, J. Chem. Phys. , 10945 (2004).

[100] D. Peng and K. Hirao, J. Chem. Phys. 130, 044102 (2009).

[101] M. Barysz, A. Sadlej, and J. G. Snijders, Int. J Quant. Chem. 65, 225 (1997).

[102] M. Barysz, J. Chem. Phys. 114, 9315 (2001).

[103] M. Barysz and A. J. Sadlej, J. Chem. Phys., 2696 (2002). 
[104] D. Kedziera and M. Barysz, J. Chem. Phys. 121, 6719 (2004).

[105] M. Iliaš, H. J. A. Jensen, V. Kellö, B. O. Roos, and M. Urban, Chem. Phys. Lett. 408, 210 (2005).

[106] W. Kutzelnigg and W. Liu, Mol. Phys. 104, 2225 (2006).

[107] W. Liu and W. Kutzelnigg, J. Chem. Phys. 126, 114107 (2007).

[108] W. Liu and D. Peng, J. Chem. Phys. 125, 044102 (2006).

[109] W. Liu and D. Peng, J. Chem. Phys. 131, 031104 (2009).

[110] H. J. A. Jensen, W. Kutzelnigg, W. Liu, T. Saue, and L. Visscher, The generic acronym X2C was introduced following discussion at the Twelfth International Conference on the Applications of Density Functional Theory (DFT-2007), Amsterdam, August 26-30, 2007.

[111] P. O. Löwdin, Adv. Phys. 5, 1 (1956).

[112] D. Peng, W. Liu, Y. Xiao, and L. Cheng, J. Chem. Phys. 127, 104106 (2007).

[113] J. Sikkema, L. Visscher, T. Saue, and M. Iliaš, J. Chem. Phys. 131, 124116 (2009).

[114] J. Seino and M. Hada, Chem. Phys. Lett. 461, 327 (2008).

[115] J. Seino and H. Nakai, "Local unitary transformation in two-component relativistic scheme for large-scale molecular systems," Poster, Sevent Congress of the International Society for Theoretical Chemical Physics (ISTCP-VII), Tokyo, Sep 2-8 2011.

[116] T. Nakajima and K. Hirao, J. Chem. Phys. 119, 4105 (2003).

[117] T. Nakajima and K. Hirao, Chem. Rev. (2011), dOI:10.1021/cr200040s.

[118] J. C. Boettger, Phys. Rev. B 62, 7809 (2000).

[119] A. Matveev and N. Rösch, J. Chem. Phys. 118, 3997 (2003).

[120] A. V. Matveev, S. Majumder, and N. Rösch, J. Chem. Phys. 123, 164104 (2005).

[121] B. A. Heß, C. M. Marian, U. Wahlgren, and O. Gropen, Chem. Phys. Lett. 251, 365 (1996).

[122] B. Schimmelpfennig, (1996), program AMFI, Stockholm, Sweden.

[123] E. J. Baerends, W. H. E. Schwarz, P. Schwerdtfeger, and J. G. Snijders, J. Phys. B: At. Mol. Opt. Phys. 23, 3225 (1990).

[124] V. Kellö and A. J. Sadlej, Int. J Quant. Chem. 68, 159 (1998).

[125] K. G. Dyall, Int. J Quant. Chem. 78, 412 (2000).

[126] M. Pernpointner and P. Schwerdtfeger, Chem. Phys. Lett. 295, 347 (1998).

[127] S. Knecht, S. Fux, R. van Meer, L. Visscher, M. Reiher, and T. Saue, Theor. Chem. Acc. 129, 631 (2011).

[128] K. G. Dyall, Theor. Chem. Acc. 112, 403 (2004), The basis sets are available from the DIRAC web site http://dirac.chem.vu.nl.

[129] K. G. Dyall and A. S. P. Gomes, Theor. Chem. Acc. 125, 97 (2010), The basis sets are available from the DIRAC web site http://dirac.chem.vu.nl.

[130] Q. Sun, W. Liu, Y. Xiao, and L. Cheng, J. Chem. Phys. 131, 081101 (2009).

[131] L. Cheng and J. Gauss, J. Chem. Phys. 135, 084114 (2011).

[132] G. Aucar, T. Saue, H. J. A. Jensen, and L. Visscher, J. Chem. Phys. 110, 6208 (1999).

[133] S. Komorovský, M. Repiský, O. L. Malkina, V. G. Malkin, I. M. Ondík, and M. Kaupp, J. Chem. Phys. 128, 104101 (2008).

[134] Y. Xiao, W. Liu, L. C., and D. Peng, J. Chem. Phys. 126, 214101 (2007).

[135] A. Antusek, M. Jaszunski, and M. Olejniczak, Comp. Theor. Chem. 970, 54 (2011).

[136] T. Fleig, Chem. Phys. (2011), DOI:10.1016/j.chemphys.2011.06.032. 